\title{
Nonlinear elliptic $p(u)$ - Laplacian problem with Fourier boundary condition
}

\author{
Stanislas Ouno $^{1}$ and Noufou Sawadogo ${ }^{2}$ \\ LAboratoire de Mathématiques et Informatique (LA.M.I) \\ 1 UFR. Sciences Exactes et Appliquées \\ Université Joseph KI ZERBO \\ 03 BP 7021 Ouaga 03, Ouagadougou, Burkina Faso \\ 2 UFR. Sciences et Techniques, \\ Université Nazi Boni, \\ 01 BP 1091 Bobo 01, Bobo-Dioulasso, Burkina Faso \\ ouaro@yahoo.fr, noufousawadogo858@yahoo.fr
}

\begin{abstract}
We study a nonlinear elliptic $p(u)$ - Laplacian problem with Fourier boundary conditions and $L^{1}-$ data. The existence and uniqueness results of entropy solutions are established.
\end{abstract}

\section{RESUMEN}

Estudiamos un problema $p(u)$-Laplaciano elíptico nolineal con condiciones de borde Fourier y datos $L^{1}$. Se establecen resultados de existencia y unicidad de soluciones de entropía.

Keywords and Phrases: variable exponent, $p(u)$-Laplacian, Young measure, Fourier boundary condition, entropy solution.

2010 AMS Mathematics Subject Classification: 35J60, 35D05, 76A05. 


\section{Introduction}

In this paper, we consider the following nonlinear Fourier boundary value problem

$$
\begin{cases}b(u)-\operatorname{div} a(x, u, \nabla u)=f & \text { in } \Omega \\ a(x, u, \nabla u) \cdot \eta+\lambda u=g & \text { on } \partial \Omega\end{cases}
$$

where $\Omega \subseteq \mathbb{R}^{N}(N \geq 3)$ is a bounded open domain with Lipschitz boundary $\partial \Omega, \eta$ is the outer unit normal vector on $\partial \Omega$ and $\lambda>0$.

The operator $\operatorname{div} a(x, u, \nabla u)$ is called $p(u)$-Laplacian. It is more complicated than $p(x)$-Laplacian in the term of nonlinearity. A prototype of this operator is $\operatorname{div}\left(|\nabla u|^{p(u)-2} \cdot \nabla u\right)$. The variable exponent $p$ depend both on the space variable $x$ and on the unknown solution $u$. The problem (1.1) is a generalization of the following nonlinear problem

$$
\begin{cases}b(u)-\operatorname{div} a(x, \nabla u)=f & \text { in } \Omega \\ a(x, \nabla u) \cdot \eta+\lambda u=g & \text { on } \partial \Omega\end{cases}
$$

studied in [15] by Nyanquini and Ouaro. The authors used an auxiliary result due to Le (see [16], Theorem 3.1) to prove the existence of the weak solution when $f \in L^{\infty}(\Omega), \quad g \in L^{\infty}(\partial \Omega)$ and by approximation methods they obtained the entropy solution when $f \in L^{1}(\Omega), g \in L^{1}(\partial \Omega)$.

In the present paper, as the function $(x, z, \eta) \mapsto a(x, z, \eta)$ is more general than $(x, \eta) \mapsto a(x, \eta)$, it is difficult to use the sub-supersolution method, as in [16, to get the existence of the weak solution. Therefore, we use the technic of pseudo-monotone operators in Banach spaces, some a priori estimates and the convergence in term of Young measure to obtain the existence of entropy solutions of problem (1.1). Indeed, we define an approximation problem, and we prove that this problem has a solution $u_{n}$ which converges to $u$, an entropy solution of problem (1.1).

In this paper, we consider the following basic assumptions on the data for the study of the problem (1.1).

$\left(A_{1}\right) \quad f$ and $g$ are some functions such as $f \in L^{1}(\Omega), g \in L^{1}(\partial \Omega)$ and $g \not \equiv 0$.

$\left(A_{2}\right) b$ is nondecreasing surjective and continuous function defined on $\mathbb{R}$ such that $b(0)=0$.

Problem (1.1) is adapted into a generalized Leray-Lions framework under the assumption that $a: \Omega \times\left(\mathbb{R} \times \mathbb{R}^{N}\right) \rightarrow \mathbb{R}^{N}$ is a Carathodory function with:

$\left(A_{3}\right) a(x, z, 0)=0$ for all $z \in \mathbb{R}$, and a.e. $x \in \Omega$

$\left(A_{4}\right)(a(x, z, \xi)-a(x, z, \eta)) \cdot(\xi-\eta)>0$ for all $\xi, \eta \in \mathbb{R}^{N}, \xi \neq \eta$, as well as the growth and the coercivity assumptions with variable exponent

$\left(A_{5}\right)|a(x, z, \xi)|^{p^{\prime}(x, z)} \leq C_{1}\left(|\xi|^{p(x, z)}+\mathcal{M}(x)\right)$ and 
$\left(A_{6}\right) a(x, z, \xi) \cdot \xi \geq \frac{1}{C_{2}}|\xi|^{p(x, z)}$

Here, $C_{1}$ and $C_{2}$ are positive constants and $\mathcal{M}$ is a positive function such that $\mathcal{M} \in L^{1}(\Omega)$.

$p: \Omega \times \mathbb{R} \rightarrow\left[p_{-}, p_{+}\right]$is a Carathodory function, $1<p_{-} \leq p_{+}<+\infty$ and $p^{\prime}(x, z)=\frac{p(x, z)}{p(x, z)-1}$ is the conjugate exponent of $p(x, z)$, with

$$
p_{-}:=e s s \inf _{(x, z) \in \Omega \times \mathbb{R}} p(x, z) \text { and } p_{+}:=e s s \sup _{(x, z) \in \Omega \times \mathbb{R}} p(x, z) \text {. }
$$

The study of $p(u)$-Laplacian problem was recently developped by Andreianov et al. (see [2]). These authors established the partial existence and uniqueness result to the weak solution in the cases of homogeneous Dirichlet boundary condition.

The interest of the study of this kind of problem is due to the fact that they can model various phenomena which arise in the study of elastic mechanic (see [6]), electrorheological fluids (see [20]) or image restoration (see [9]).

In this paper, we study the existence of the weak solution for approximation problem and we also establish the existence and uniqueness results of the entropy solution when the data are in $L^{1}$.

In this work, we use the Sobolev embedding results and the convergence in term of Young measure (see [10, 12]).

Here, we consider a Fourier boundary condition which bring some difficulties to treat the term at the boundary.

We were inspired by the work of Ouaro and Tchousso (see [15]), where the authors defined for the first time a new space by taking into account the boundary.

For the next part of the paper (section 2), we introduce some preliminary results. In section 3, we study the existence and uniqueness of entropy solution when the data are in $L^{1}$.

\section{Preliminary}

- We will use the so-called truncation function

$$
T_{k}(s):=\left\{\begin{array}{ll}
s & \text { if }|s| \leq k \\
\operatorname{ksign}_{0}(s) & \text { if }|s|>k
\end{array}, \quad \text { where } \operatorname{sign}_{0}(s):= \begin{cases}1 & \text { if } s>0 \\
0 & \text { if } s=0 \\
-1 & \text { if } s<0 .\end{cases}\right.
$$

The truncation function possesses the following properties.

$$
\begin{aligned}
T_{k}(-s) & =-T_{k}(s),\left|T_{k}(s)\right|=\min \{|s|, k\}, \\
\lim _{k \rightarrow+\infty} T_{k}(s) & =s \text { and } \lim _{k \rightarrow 0} \frac{1}{k} T_{k}(s)=\operatorname{sign}_{0}(s) .
\end{aligned}
$$


We also need to truncate vector valued-function with the help of the mapping

$$
h_{m}: \mathbb{R}^{N} \longrightarrow \mathbb{R}^{N}, h_{m}(\lambda)=\left\{\begin{array}{ll}
\lambda, & \text { if }|\lambda| \leq m \\
m \frac{\lambda}{|\lambda|} & \text { if }|\lambda|>m,
\end{array} \text { where } m>0 .\right.
$$

For a Lebesgue measurable set $A \subset \Omega, \chi_{A}$ denotes its characteristic function and meas $(A)$ denotes its Lebesgue measure. Let $u: \Omega \rightarrow \mathbb{R}$ be a function and $k \in \mathbb{R}$, we write $\{|u| \leq k\}$ or $[|u| \leq k]$ for the set $\{x \in \Omega:|u(x)| \leq k\}$, (respectively, $\geq,=,<,>$ ).

The function $a(., .,$.$) appearing in (1.1) satisfies a generalized Leray-Lions assumptions given in$ Introduction. View that, $a(., .,$.$) satisfies \left(A_{5}\right)$ and $\left(A_{6}\right)$, we must work in Lebesgue and Sobolev spaces with variable exponent, that depend on $x$ and on $u(x)$. For the study of problem (1.1), we need the Sobolev spaces $W^{1, \pi(.)}(\Omega)$, where $\pi()=.p(., u()$.$) .$

Definition 1. Let $\pi: \Omega \longrightarrow[1,+\infty)$ be a measurable function for $\pi()=.p(., u()$.$) .$ - $L^{\pi(.)}(\Omega)$ is the space of all measurable function $f: \Omega \longrightarrow \mathbb{R}$ such that the modular

$$
\rho_{\pi(.)}(f):=\int_{\Omega}|f|^{\pi(x)} d x<+\infty .
$$

If $p_{+}$is finite, this space is equipped with the Luxembourg norm

$$
\|f\|_{L^{\pi(.)}(\Omega)}:=\inf \left\{\lambda>0 ; \quad \rho_{\pi(.)}\left(\frac{f}{\lambda}\right) \leq 1\right\} .
$$

In the sequel, we will use the same notation $L^{\pi(.)}(\Omega)$ for the space $\left(L^{\pi(.)}(\Omega)\right)^{N}$ of vector-valued functions.

- $W^{1, \pi(.)}(\Omega)$ is the space of all functions $f \in L^{\pi(\cdot)}(\Omega)$ such that the gradient of $f$ (taken in the sense of distributions) belongs to $L^{\pi(\cdot)}(\Omega)$. The space $W^{1, \pi(\cdot)}(\Omega)$ is equipped with the norm

$$
\|u\|_{W^{1, \pi(\cdot)}(\Omega)}:=\|u\|_{L^{\pi(\cdot)}(\Omega)}+\|\nabla u\|_{L^{\pi(\cdot)}(\Omega)} .
$$

When $1<p_{-} \leq \pi(.) \leq p_{+}<+\infty$, all the above spaces are separable and reflexive Banach spaces.

We denote $\pi_{n}(x):=p\left(x, u_{n}(x)\right)$.

Proposition 1. (See [1], Proposition 2.3)

For all measurable function $\pi: \Omega \rightarrow\left[p_{-}, p_{+}\right]$, the following properties hold.

i) $L^{\pi(\cdot)}(\Omega)$ and $W^{1, \pi(\cdot)}(\Omega)$ are separable and reflexive Banach spaces.

ii) $L^{\pi^{\prime}(.)}(\Omega)$ can be identified with the dual space of $L^{\pi(.)}(\Omega)$, and the following Hlder type inequality holds:

$$
\forall f \in L^{\pi(\cdot)}(\Omega), g \in L^{\pi^{\prime}(\cdot)}(\Omega), \quad\left|\int_{\Omega} f g d x\right| \leq 2|| f\left\|_{L^{\pi(\cdot)}(\Omega)}\right\| g \|_{L^{\pi^{\prime}(\cdot)}(\Omega)} .
$$


iii) One has $\rho_{\pi(.)}(f)=1$ if and only if $\|f\|_{L^{\pi(.)}(\Omega)}=1$; further,

$$
\begin{aligned}
& \text { if } \rho_{\pi(.)}(f) \leq 1 \text {, then }\|f\|_{L^{\pi(.)}(\Omega)}^{p_{+}} \leq \rho_{\pi(.)}(f) \leq\|f\|_{L^{\pi(.)}(\Omega)}^{p_{-}} \\
& \text {if } \rho_{\pi^{(.)}}(f) \geq 1 \text {, then }\|f\|_{L^{\pi(.)}(\Omega)}^{p_{-}} \leq \rho_{\pi(.)}(f) \leq\|f\|_{L^{\pi(.)}(\Omega)}^{p_{+}} \text {. }
\end{aligned}
$$

In particular, if $\left(f_{n}\right)_{n \in \mathbb{N}}$ is a sequence in $L^{\pi(.)}(\Omega)$, then $\left\|f_{n}\right\|_{L^{\pi(.)}(\Omega)}$ tends to zero (resp., to infinity) if and only if $\rho_{\pi(.)}\left(f_{n}\right)$ tends to zero (resp., to infinity), as $n \rightarrow+\infty$.

For a measurable function $f \in W^{1, \pi(.)}(\Omega)$ we introduce the following notation:

$$
\rho_{1, \pi(.)}(f)=\int_{\Omega}|f|^{\pi(.)} d x+\int_{\Omega}|\nabla f|^{\pi(.)} d x .
$$

Replacing $p(x)$ by $\pi(x)$ in [8]-Proposition 2.2, we obtain the following result that is fundamental in this paper.

Proposition 2. ( See [23, 24] ) If $f \in W^{1, \pi(.)}(\Omega)$, the following properties hold:
i) $\|f\|_{W^{1, \pi(\cdot)}(\Omega)}>1 \Rightarrow\|f\|_{W^{1, \pi(.)}(\Omega)}^{p_{-}}<\rho_{1, \pi(.)}(f)<\|f\|_{W^{1, \pi(.)}(\Omega)}^{p_{+}}$;
ii) $\|f\|_{W^{1, \pi(.)}(\Omega)}<1 \Rightarrow\|f\|_{W^{1, \pi(.)}(\Omega)}^{p_{+}}<\rho_{1, \pi(.)}(f)<\|f\|_{W^{1, \pi(.)}(\Omega)}^{p_{-}} ;$
iii) $\|f\|_{W^{1, \pi(.)}(\Omega)}<1 \quad$ (respectively $\left.=1 ;>1\right) \Leftrightarrow \rho_{1, \pi(.)}(f)<1 \quad$ (respectively $=1 ;>1$ ).

The following lemma prove that the space $W^{1, \pi(.)}(\Omega)$ is stable by truncation.

Lemma 2.1. If $u \in W^{1, \pi(.)}(\Omega)$ then $T_{k}(u) \in W^{1, \pi(.)}(\Omega)$.

Now, we give the embedding results.

Proposition 3. (See [1], Proposition 2.4) Assume that $\pi: \Omega \rightarrow\left[p_{-}, p_{+}\right]$has a representative which can be extended into a continuous function up to the boundary $\partial \Omega$ and satisfying the logHlder continuity assumption:

$$
\exists L>0, \quad \forall x, y \in \bar{\Omega}, x \neq y, \quad-(\log |x-y|)|\pi(x)-\pi(y)| \leq L .
$$

i) Then, $\mathcal{D}(\Omega)$ is dense in $W^{1, \pi(\cdot)}(\Omega)$.

ii) $W^{1, \pi(.)}(\Omega)$ is embedded into $L^{\pi^{*}(.)}(\Omega)$, where $\pi^{*}($.$) is the Sobolev embedding exponent defined$ as in (2.2) below. If $q$ is a measurable variable exponent such that ess $\inf _{x \in \Omega}\left(\pi^{*}()-.q().\right)>0$, then the embedding of $W^{1, \pi(\cdot)}(\Omega)$ into $L^{q(.)}(\Omega)$ is compact. 
For a given $\pi($.$) , a function taking values in \left[p_{-}, p_{+}\right], \pi^{*}($.$) denotes the optimal Sobolev embedding$ defined for any $x \in \Omega$ by

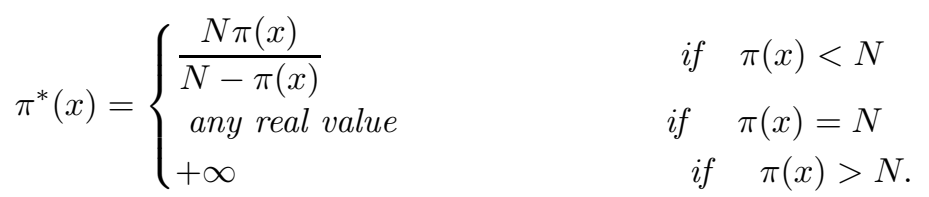

Put

$$
\pi^{\partial}(x):=(\pi(x))^{\partial}:=\left\{\begin{array}{lr}
\frac{(N-1) \pi(x)}{N-\pi(x)} & \text { if } \quad \pi(x)<N \\
+\infty & \text { if } \quad \pi(x) \geq N .
\end{array}\right.
$$

Proposition 4. (See [18], Proposition 2.3)

Let $\pi(.) \in C(\bar{\Omega})$ and $p_{-}>1$. If $q(x) \in C(\partial \Omega)$ satisfies the condition:

$$
1 \leq q(x)<\pi^{\partial}(x), \quad \forall x \in \partial \Omega,
$$

then, there is a compact embedding

$$
W^{1, \pi(\cdot)}(\Omega) \hookrightarrow L^{q(\cdot)}(\partial \Omega) .
$$

In particular there is compact embedding

$$
W^{1, \pi(\cdot)}(\Omega) \hookrightarrow L^{\pi(\cdot)}(\partial \Omega) .
$$

\section{TYoung measures and nonlinear weak-* convergence.}

Throughout the paper, we denote by $\delta_{c}$ the Dirac measure on $\mathbb{R}^{d}(d \in \mathbb{N})$, concentrated at the point $c \in \mathbb{R}^{d}$.

In the following theorem, we gather the results of Ball [7, Pedregal [19] and Hungerbühler [13] which will be needed for our purposes (we limit the statement to the case of a bounded domain $\Omega$ ). Let us underline that the results of (ii),(iii), expressed in terms of the convergence in measure, are very convenient for the applications that we have in mind.

Theorem 2.1. (i) Let $\Omega \subset \mathbb{R}^{N}, N \in \mathbb{N}$, and a sequence $\left(v_{n}\right)_{n \in \mathbb{N}}$ of $\mathbb{R}^{d}$-valued functions, $d \in \mathbb{N}$ , such that $\left(v_{n}\right)_{n \in \mathbb{N}}$ is equi-integrable on $\Omega$. Then, there exists a subsequence $\left(n_{k}\right)_{k \in \mathbb{N}}$ and a parametrized family $\left(\nu_{x}\right)_{x \in \Omega}$ of probability measures on $\mathbb{R}^{d}(d \in \mathbb{N})$, weakly measurable in $x$ with respect to the Lebesgue measure in $\Omega$, such that for all Carathodory function $F$ : $\Omega \times \mathbb{R}^{d} \rightarrow \mathbb{R}^{t}, t \in \mathbb{N}$, we have

$$
\lim _{k \rightarrow+\infty} \int_{\Omega} F\left(x, v_{n_{k}}\right) d x=\int_{\Omega} \int_{\mathbb{R}^{d}} F(x, \lambda) d \nu_{x}(\lambda) d x,
$$


whenever the sequence $\left(F\left(., v_{n}(.)\right)\right)_{n \in \mathbb{N}}$ is equi-integrable on $\Omega$. In particular,

$$
v(x):=\int_{\mathbb{R}^{d}} \lambda d \nu_{x}(\lambda)
$$

is the weak limit of the sequence $\left(v_{n_{k}}\right)_{k \in \mathbb{N}}$ in $L^{1}(\Omega)$.

The family $\left(\nu_{x}\right)_{x \in \Omega}$ is called the Young measure generated by the subsequence $\left(v_{n_{k}}\right)_{k \in \mathbb{N}}$.

(ii) If $\Omega$ is of finite measure, and $\left(\nu_{x}\right)_{x \in \Omega}$ is the Young measure generated by a sequence $\left(v_{n}\right)_{n \in \mathbb{N}}$, then $\nu_{x}=\delta_{v(x)}$ for a.e. $x \in \Omega \Leftrightarrow v_{n}$ converges in measure in $\Omega$ to $v$ as $n \rightarrow+\infty$.

(iii) If $\Omega$ is of finite measure, $\left(u_{n}\right)_{n \in \mathbb{N}}$ generates a Dirac Young measure $\left(\delta_{u(x)}\right)_{x \in \Omega}$ on $\mathbb{R}^{d_{1}}$, and $\left(v_{n}\right)_{n \in \mathbb{N}}$ generates a Young measure $\left(\nu_{x}\right)_{x \in \Omega}$ on $\mathbb{R}^{d_{2}}$, then the sequence $\left(u_{n}, v_{n}\right)_{n \in \mathbb{N}}$ generates the Young measure $\left(\delta_{u(x)} \otimes \nu_{x}\right)_{x \in \Omega}$ on $\mathbb{R}^{d_{1}+d_{2}}$. Whenever a sequence $\left(v_{n}\right)_{n \in \mathbb{N}}$ generates a Young measure $\left(\nu_{x}\right)_{x \in \Omega}$, following the terminology of [11] we will say that $\left(v_{n}\right)_{n \in \mathbb{N}}$ nonlinear weak-* converges, and $\left(\nu_{x}\right)_{x \in \Omega}$ is the nonlinear weak-* limit of the sequence $\left(v_{n}\right)_{n \in \mathbb{N}}$. In the case where $\left(v_{n}\right)_{n \in \mathbb{N}}$ possesses a nonlinear weak- ${ }^{*}$ convergent subsequence, we will say that it is nonlinear weak-* compact. ([1], Theorem 2.10(i)) It means that any equi-integrable sequence of measurable functions is nonlinear weak-* compact on $\Omega$.

Lemma 2.2. (See [1], Theorem 3.11 and [2] Step 2 of proof of Theorem 2.6). Assume that $\left(u_{n}\right)_{n \in \mathbb{N}}$ converges a.e. on $\Omega$ to some function $u$, then

$$
\begin{aligned}
& \left|p\left(x, u_{n}(x)\right)-p(x, u(x))\right| \text { converges in measure to } 0 \text { on } \Omega, \\
& \text { and for all bounded subset } K \text { of } \mathbb{R}^{N}, \\
& \sup _{\xi \in K}\left|a\left(x, u_{n}(x), \xi\right)-a(x, u(x), \xi)\right| \quad \text { converges in measure to } 0 \text { on } \Omega .
\end{aligned}
$$

For the sequel, we assume that $p(.,$.$) is \log$ Hlder continuous uniformly on $\bar{\Omega} \times[-M, M]$ and $p_{-}>N$. We recall some notations.

For any $u \in W^{1, \pi(.)}(\Omega)$, we denote by $\tau(u)$ the trace of $u$ on $\partial \Omega$ in the usual sense.

We will identify at boundary $u$ and $\tau(u)$.

Set

$$
\mathcal{T}^{1, \pi(.)}(\Omega)=\left\{u: \Omega \rightarrow \mathbb{R}, \text { measurable such that } T_{k}(u) \in W^{1, \pi(.)}(\Omega) \text {, for any } k>0\right\} .
$$

\section{Entropy solution}

In this part, we study the existence and uniqueness of the entropy solution to the problem (1.1). We give some notations. 
We define $\mathcal{T}_{t r}^{1, \pi(.)}(\Omega)$ as the set of the functions $u \in \mathcal{T}^{1, \pi(.)}(\Omega)$ such that there exists a sequence $\left(u_{n}\right)_{n \in \mathbb{N}} \subset W^{1, p_{+}}(\Omega)$ satisfying the following conditions:

$\left(C_{1}\right) u_{n} \rightarrow u$ a.e. in $\Omega$.

$\left(C_{2}\right) \nabla T_{k}\left(u_{n}\right) \rightarrow \nabla T_{k}(u)$ in $L^{1}(\Omega)$.

$\left(C_{3}\right)$ There exists a measurable function $v$ on $\partial \Omega$, such that $u_{n} \rightarrow v$ a.e. on $\partial \Omega$.

The function $v$ is the trace of $u$ in the generalized sense as introduced in [4, 5]. In the sequel the trace of $u \in \mathcal{T}_{t r}^{1, \pi(.)}(\Omega)$ on $\partial \Omega$ will be denoted $\operatorname{tr}(u)$. If $u \in W^{1, \pi(.)}(\Omega), \operatorname{tr}(u)$ coincides with $\tau(u)$ in the usual sense. Moreover, for $u \in \mathcal{T}_{t r}^{1, \pi(.)}(\Omega)$ and for all $k>0, \operatorname{tr}\left(T_{k}(u)\right)=T_{k}(\operatorname{tr}(u))$ and if $\varphi \in W^{1, \pi(.)}(\Omega)$ then $u-\varphi \in \mathcal{T}_{t r}^{1, \pi(.)}(\Omega)$ and $\operatorname{tr}(u-\varphi)=\operatorname{tr}(u)-\operatorname{tr}(\varphi)$.

As in [1-Proposition 3.5, we give the following result.

Proposition 5. Let $u \in \mathcal{T}^{1, \pi(.)}(\Omega)$. There exists a unique measurable function $w: \Omega \rightarrow \mathbb{R}^{N}$ such that $\nabla T_{k}(u)=w \chi_{\{|u|<k\}}$ for $k>0$. The function $w$ is denoted by $\nabla u$. Moreover, if $u \in W^{1, \pi(.)}(\Omega)$ then $w \in L^{\pi(.)}(\Omega)$ and $w=\nabla u$ in the usual sense.

Remark 3.1. The space $\mathcal{T}_{\text {tr }}^{1, \pi(.)}(\Omega)$ in our context will be a subset of $\mathcal{T}^{1, \pi(.)}(\Omega)$ consisting to the function can be approximated by function of $W^{1, p_{+}}(\Omega)$. Since the weak solution of approximated problem (3.2) belongs to $W^{1, p_{+}}(\Omega)$.

Now, we introduce the notion of entropy solution due to Ouaro and al. [14, Definition 3.1].

Definition 2. A measurable function $u: \Omega \rightarrow \mathbb{R}$ for $\pi()=.p(., u()$.$) is called entropy solution of$ the problem (1.1) if

$$
u \in \mathcal{T}_{t r}^{1, \pi(\cdot)}(\Omega), \quad b(u) \in L^{1}(\Omega), \quad u \in L^{1}(\partial \Omega)
$$

and for all $k>0$,

$$
\begin{aligned}
\int_{\Omega} b(u) T_{k}(u-\varphi) d x+\int_{\Omega} a(x, u, \nabla u) \cdot \nabla T_{k}(u-\varphi) d x & +\lambda \int_{\partial \Omega} u T_{k}(u-\varphi) d \sigma \\
& \leq \int_{\Omega} f T_{k}(u-\varphi) d x+\int_{\partial \Omega} g T_{k}(u-\varphi) d \sigma
\end{aligned}
$$

where $\varphi \in W^{1, \pi(.)}(\Omega) \cap L^{\infty}(\Omega)$.

The following theorem gives existence result of entropy solution.

Theorem 3.2. Assume that $\left(A_{3}\right)-\left(A_{6}\right)$ hold and $f \in L^{1}(\Omega), g \in L^{1}(\partial \Omega)$. Then, there exists at least one entropy solution to the problem (1.1).

The proof of Theorem 3.2 is done in two parts. 


\section{Part 1: The approximate problem.}

Let $f_{n}=T_{n}(f)$ and $g_{n}=T_{n}(g)$. Then, $f_{n} \in L^{\infty}(\Omega)$ and $g_{n} \in L^{\infty}(\partial \Omega)$. Moreover, $\left(f_{n}\right)_{n \in \mathbb{N}}$ strongly converges to $f$ in $L^{1}(\Omega)$ and $\left(g_{n}\right)_{n \in \mathbb{N}}$ strongly converges to $g$ in $L^{1}(\partial \Omega)$ such that $\left\|f_{n}\right\|_{L^{1}(\Omega)} \leq$ $\|f\|_{L^{1}(\Omega)}$ and $\left\|g_{n}\right\|_{L^{1}(\partial \Omega)} \leq\|g\|_{L^{1}(\partial \Omega)}$.

We consider the following problem

$$
\begin{cases}T_{n}\left(b\left(u_{n}\right)\right)-\operatorname{div} a\left(x, u_{n}, \nabla u_{n}\right)-\varepsilon \triangle_{p_{+}} u_{n}+\varepsilon\left|u_{n}\right|^{p_{+}-2} u_{n}=f_{n} & \text { in } \quad \Omega \\ \left(a\left(x, u_{n}, \nabla u_{n}\right)+\varepsilon\left|\nabla u_{n}\right|^{p_{+}-2} \nabla u_{n}\right) \cdot \eta+\lambda T_{n}\left(u_{n}\right)=g_{n} & \text { on } \quad \partial \Omega,\end{cases}
$$

where

$$
-\triangle_{p_{+}} u_{n}:=-\sum_{i=1}^{N} \frac{\partial}{\partial x_{i}}\left(\left|\frac{\partial u_{n}}{\partial x_{i}}\right|^{p_{+}-2} \frac{\partial u_{n}}{\partial x_{i}}\right) .
$$

In this part, we show that the problem (3.2) admits at least one weak solution $u_{n}$, for all $\varepsilon>0$. We define the following reflexive space

$$
E=W^{1, p_{+}}(\Omega) \times L^{p_{+}}(\partial \Omega)
$$

Let

$$
X_{0}=\{(u, v) \in E: \quad v=\tau(u)\} .
$$

In the sequel, we will identify an element $(u, v) \in X_{0}$ with its representative $u \in W^{1, p_{+}}(\Omega)$ (since $\left.W^{1, p_{+}}(\Omega) \hookrightarrow \hookrightarrow L^{p_{+}}(\partial \Omega)\right)$.

Theorem 3.3. There exists at least one weak solution $u_{n}$ for the problem (3.2) in the sense that $u_{n} \in X_{0}$ and for all $v \in X_{0}$,

$$
\begin{aligned}
\int_{\Omega} T_{n}\left(b\left(u_{n}\right)\right) v d x+\int_{\Omega} a\left(x, u_{n}, \nabla u_{n}\right) \nabla v d x & +\int_{\partial \Omega} \lambda T_{n}\left(u_{n}\right) v d \sigma \\
& +\varepsilon \int_{\Omega}\left[\left|u_{n}\right|^{p_{+}-2} u_{n} v+\left|\nabla u_{n}\right|^{p_{+}-2} \nabla u_{n} \nabla v\right] d x \\
& =\int_{\Omega} f_{n} v d x+\int_{\partial \Omega} g_{n} v d \sigma .
\end{aligned}
$$

To prove the Theorem 3.3 we need the following result.

Lemma 3.1. (See [22], Corollary 2.2). If an operator $\mathcal{A}$ is of type $(M)$, bounded and coercive on a separable Banach space to its dual, then $\mathcal{A}$ is surjective.

We define the operator $A_{n}$ by

$$
A_{n} u=A u+B_{n} u
$$


where

$$
<A u, v>=\int_{\Omega} a(x, u, \nabla u) \nabla v d x
$$

and

$$
<B_{n} u, v>=\int_{\Omega} T_{n}(b(u)) v d x+\lambda \int_{\partial \Omega} T_{n}(u) v d \sigma+\varepsilon \int_{\Omega}\left[|u|^{p_{+}-2} u v+|\nabla u|^{p_{+}-2} \nabla u \nabla v\right] d x,
$$

with $u, v \in X_{0}$.

Proof of the Theorem 3.3 . The proof is organized in three Steps.

Step 1: $A_{n}$ is bounded.

By using Hlder type inequality and $\left(A_{5}\right)$ with constant exponent $p_{+}$, we deduce that $A$ is bounded.

Moreover, $B_{n}$ is bounded. Indeed, let $u \in F$, where $F$ is a bounded subset of $X_{0}$.

As $b$ is onto, we have

$$
\begin{aligned}
<B_{n} u, v> & =\int_{\Omega} T_{n}(b(u)) v d x+\lambda \int_{\partial \Omega} T_{n}(u) v d \sigma+\varepsilon \int_{\Omega}\left[|u|^{p_{+}-2} u v+|\nabla u|^{p_{+}-2} \nabla u \nabla v\right] d x \\
& \leq \int_{\Omega}\left|b(u)\left\|v\left|d x+\lambda \int_{\partial \Omega}\right| u\right\| v\right| d \sigma+\varepsilon \int_{\Omega}\left[|u|^{p_{+}-1}|v|+|\nabla u|^{p_{+}-1}|\nabla v|\right] d x \\
& \leq C(\lambda)\left(\|v\|_{L^{1}(\Omega)}+\|v\|_{L^{1}(\partial \Omega)}\right)+\varepsilon\left[\|u\|_{\left.L^{p_{+}(\Omega)}\right)}^{\frac{p_{+}}{\left(p_{+}^{\prime}\right.}}\|v\|_{L^{p_{+}}(\Omega)}+\|\nabla u\|_{L^{p_{+}}(\Omega)}^{\frac{p_{+}}{\left(p_{+}^{\prime}\right.}}\|\nabla v\|_{L^{p_{+}(\Omega)}}\right] \\
& \leq C(\lambda)\left(\|v\|_{L^{1}(\Omega)}+\|v\|_{L^{1}(\partial \Omega)}\right)+C(\varepsilon)\|v\|_{W^{1, p_{+}(\Omega)}} .
\end{aligned}
$$

Therefore, $A_{n}$ is bounded.

We recall the following notion:

Definition 3. An operator $A: V \rightarrow V^{\prime}$ is type of $(M)$ if:

$$
\left.\begin{array}{l}
u_{n} \rightarrow u \text { in } V \\
A\left(u_{n}\right) \rightarrow \chi \text { in } V^{\prime} \\
\limsup _{n \rightarrow \infty}<A\left(u_{n}\right), u_{n}>\leq<\chi, u>
\end{array}\right\} \Rightarrow \chi=A(u) .
$$

Step 2: $A_{n}$ is pseudo-monotone.

Let $\left(u_{k}\right)_{k \in \mathbb{N}}$ be a sequence in $X_{0}$ such that

$$
\left\{\begin{array}{l}
u_{k} \rightarrow u \text { in } X_{0} \\
A_{n} u_{k} \rightarrow \chi \text { in } X_{0}^{\prime} \\
\limsup _{k \rightarrow \infty}<A_{n} u_{k}, u_{k}>=<\chi, u>.
\end{array}\right.
$$

We will prove that $\chi=A_{n} u$.

As $T_{n}\left(b\left(u_{k}\right)\right) u_{k} \geq 0$ and $\lambda T_{n}\left(u_{k}\right) u_{k} \geq 0$, by Fatou's Lemma, we deduce that

$$
\liminf _{k \rightarrow \infty}\left(\int_{\Omega} T_{n}\left(b\left(u_{k}\right)\right) u_{k} d x+\lambda \int_{\partial \Omega} T_{n}\left(u_{k}\right) u_{k} d \sigma\right) \geq \int_{\Omega} T_{n}(b(u)) u d x+\lambda \int_{\partial \Omega} T_{n}(u) u d \sigma .
$$


One the other hand, thanks to the Lebesgue dominated convergence Theorem, we have

$$
\begin{array}{r}
\lim _{k \rightarrow \infty}\left(\int_{\Omega} T_{n}\left(b\left(u_{k}\right)\right) v d x+\lambda \int_{\partial \Omega} T_{n}\left(u_{k}\right) v d \sigma+\varepsilon \int_{\Omega}\left[\left|u_{k}\right|^{p_{+}-2} u_{k} v+\left|\nabla u_{k}\right|^{p_{+}-2} \nabla u_{k} \nabla v\right] d x\right) \\
=\int_{\Omega} T_{n}(b(u)) v d x+\lambda \int_{\partial \Omega} T_{n}(u) v d \sigma+\varepsilon \int_{\Omega}\left[|u|^{p_{+}-2} u v+|\nabla u|^{p_{+}-2} \nabla u \nabla v\right] d x
\end{array}
$$

for any $v \in X_{0}$. Therefore, for $k$ large enough,

$T_{n}\left(b\left(u_{k}\right)\right)+\lambda T_{n}\left(u_{k}\right)+\varepsilon\left[\left|u_{k}\right|^{p_{+}-2} u_{k}+\left|\nabla u_{k}\right|^{p_{+}-2} \nabla u_{k}\right] \rightarrow T_{n}(b(u))+\lambda T_{n}(u)+\varepsilon\left[|u|^{p_{+}-2} u+|\nabla u|^{p_{+}-2} \nabla u\right]$ in $X_{0}^{\prime}$.

Thus,

$$
A u_{k} \rightarrow \chi-\left(T_{n}(b(u))+\lambda T_{n}(u)+\varepsilon\left[|u|^{p_{+}-2} u+|\nabla u|^{p_{+}-2} \nabla u\right]\right) \text { in } X_{0}^{\prime}, \text { as } k \rightarrow+\infty
$$

Now, we are going to prove that $A$ is of type $(M)$.

Let us set

$$
a_{1}(u, v, w)=\int_{\Omega} a(x, u, \nabla v) \nabla w d x
$$

Then, $w \mapsto a_{1}(u, v, w)$ is continuous on $W^{1, p_{+}}(\Omega)$, thus

$$
a_{1}(u, v, w)=\langle A(u, v), w\rangle, \quad A(u, v) \in\left(W^{1, p_{+}}(\Omega)\right)^{\prime},
$$

and verify

$$
A(u, u)=A u, \text { where } A u:=-\operatorname{div} a(x, u, \nabla u) .
$$

\section{Let us prove that $A$ is of type of Calculus of variation.}

- As $A(u,$.$) is bounded, we prove that v \mapsto A(u, v)$ is hemi-continuous from $W^{1, p_{+}}(\Omega) \rightarrow\left(W^{1, p_{+}}(\Omega)\right)^{\prime}$. Since $a\left(x, u, \nabla\left(v_{1}+t v_{2}\right)\right) \rightarrow a\left(x, u, \nabla v_{1}\right)$ in $L^{p_{+}^{\prime}}(\Omega)$ as $t \rightarrow 0$ and $u, v_{1}, v_{2} \in W^{1, p_{+}}(\Omega)$ then, $a_{1}\left(u, v_{1}+t v_{2}, w\right) \rightarrow a_{1}\left(u, v_{1}, w\right)$ as $t \rightarrow 0$.

In the same manner we prove that $u \mapsto A(u, v)$ is hemi-continuous from $W^{1, p_{+}}(\Omega) \rightarrow\left(W^{1, p_{+}}(\Omega)\right)^{\prime}$.

Moreover, for all $u, v \in W^{1, p_{+}}(\Omega)$, we have

$$
\begin{aligned}
<A(u, u)-A(u, v), u-v\rangle & =\langle A(u, u), u-v\rangle-\langle A(u, v), u-v\rangle \\
& =a_{1}(u, u, u-v)-a_{1}(u, v, u-v) \\
& =\int_{\Omega} a(x, u, \nabla u) \nabla(u-v) d x-\int_{\Omega} a(x, u, \nabla v) \nabla(u-v) d x \\
& =\int_{\Omega}(a(x, u, \nabla u)-a(x, u, \nabla v)) \nabla(u-v) d x \geq 0 .
\end{aligned}
$$


- Let us suppose that $u_{k} \rightarrow u$ in $W^{1, p_{+}}(\Omega)$ and $<A\left(u_{k}, u_{k}\right)-A\left(u_{k}, u\right), u_{k}-u>\rightarrow 0$. We prove that

$$
\forall v \in W^{1, p_{+}}(\Omega), \quad A\left(u_{k}, v\right) \rightarrow A(u, v) \text { in }\left(W^{1, p_{+}}(\Omega)\right)^{\prime}
$$

Let's set

$$
\int_{\Omega} F_{k} d x=\left\langle A\left(u_{k}, u_{k}\right)-A\left(u_{k}, u\right), u_{k}-u\right\rangle, \text { then } F_{k} \rightarrow 0 .
$$

As $u_{k} \rightarrow u$, we have

$$
a\left(x, u_{k}, \nabla v\right) \rightarrow a(x, u, \nabla v) \text { in } L^{p_{+}^{\prime}}(\Omega)
$$

(see [17], Lemma 2.2 with $m=1)$. Therefore, $A\left(u_{k}, v\right) \rightarrow A(u, v)$ in $\left(W^{1, p_{+}}(\Omega)\right)^{\prime}$.

- Now, we suppose that $u_{k} \rightarrow u$ in $W^{1, p_{+}}(\Omega)$ and $A\left(u_{k}, v\right) \rightarrow \Theta$ in $\left(W^{1, p_{+}}(\Omega)\right)^{\prime}$. We prove that

$$
\left\langle A\left(u_{k}, v\right), u_{k}\right\rangle \rightarrow\langle\Theta, u\rangle \text {. }
$$

Then, by using ([17, Lemma 2.1), we obtain that $a\left(x, u_{k}, \nabla v\right) \rightarrow a(x, u, \nabla v)$ in $L^{p_{+}^{\prime}}(\Omega)$ and thus, $a_{1}\left(u_{k}, v, u_{k}\right) \rightarrow a_{1}(u, v, u)$.

Therefore,

$$
<A\left(u_{k}, v\right), u_{k}>=a_{1}\left(u_{k}, v, u_{k}\right) \rightarrow<A(u, v), u>\text { and } \Theta=A(u, v) .
$$

Hence, $A$ is of type of Calculus of variation. Finally, by using ([17, Proposition 2.6 and Proposition $2.5)$, we prove that $A$ is of type $(M)$.

As the operator $A$ is of type $(M)$, so we have immediately

$$
A u=\chi-\left(T_{n}(b(u))+\lambda T_{n}(u)+\varepsilon\left[|u|^{p_{+}-2} u+|\nabla u|^{p_{+}-2} \nabla u\right]\right) .
$$

Therefore, we deduce that $A_{n} u=\chi$.

Step 3: $A_{n}$ is coercive.

$$
\begin{aligned}
<A_{n} u, u> & =\int_{\Omega} a(x, u, \nabla u) \cdot \nabla u d x+\int_{\Omega} T_{n}(b(u)) u d x \\
& +\lambda \int_{\partial \Omega} T_{n}(u) u d x+\varepsilon \int_{\Omega}\left[|u|^{p_{+}}+|\nabla u|^{p_{+}}\right] d x \\
& \geq \varepsilon \int_{\Omega}\left[|u|^{p_{+}}+|\nabla u|^{p_{+}}\right] d x \\
& \geq \varepsilon\|u\|_{W^{1, p_{+}(\Omega)}}^{p_{+}} .
\end{aligned}
$$

We deduce that

$$
\frac{<A_{n} u, u>}{\|u\|_{W^{1, p}+(\Omega)}} \rightarrow+\infty \quad \text { as }\|u\|_{W^{1, p}(\Omega)} \rightarrow+\infty
$$


Hence, $A_{n}$ is coercive.

Then, according to Lemma 3.1, $A_{n}$ is surjective.

Thus, for any $F_{n}=<T_{n}(f), T_{n}(g)>\subset E^{\prime} \subset X_{0}^{\prime}$, there exists at least one solution $u_{n} \in X_{0}$ of the problem

$$
<A_{n} u_{n}, v>=<F_{n}, v>\text { for all } v \in X_{0} .
$$

Therefore, $u_{n}$ is a weak solution of the problem (3.2). This ends the proof of Theorem 3.3 ,

Remark 3.4. If $u_{n}$ is a weak solution of the problem (3.2), then $u_{n} \in W^{1, \pi_{n}(.)}(\Omega)$, since $W^{1, p_{+}}(\Omega) \hookrightarrow$ $W^{1, \pi_{n}(.)}(\Omega)$ continuously. Moreover, $a\left(x, u_{n}, \nabla u_{n}\right)$ satisfies $\left(A_{3}\right)-\left(A_{6}\right)$ with variable exponent $\pi_{n}(x):=p\left(x, u_{n}(x)\right)$.

\section{Part 2: A priori estimates and convergence results.}

This part is done in three steps, we make a priori estimates, some convergence results and other based on the Young measure and nonlinear weak $-{ }^{*}$ convergence.

\section{Step 1: A priori estimates}

Lemma 3.2. Suppose that $\left(A_{3}\right)-\left(A_{6}\right)$ hold with variable exponent $\pi_{n}($.$) and f_{n} \in L^{\infty}(\Omega), g_{n} \in$ $L^{\infty}(\partial \Omega)$. Let $u_{n}$ be a weak solution of (3.2). Then, for all $k>0$,

$$
\begin{gathered}
\int_{\Omega}\left|\nabla T_{k}\left(u_{n}\right)\right|^{\pi_{n}(.)} d x \leq C_{2} k\left(\|f\|_{L^{1}(\Omega)}+\|g\|_{L^{1}(\partial \Omega)}\right), \\
\int_{\Omega}\left|T_{n}\left(b\left(u_{n}\right)\right)\right| d x \leq\|f\|_{L^{1}(\Omega)}+\|g\|_{L^{1}(\partial \Omega)}, \\
\int_{\partial \Omega}\left|T_{n}\left(u_{n}\right)\right| d x \leq \frac{1}{\lambda}\left(\|f\|_{L^{1}(\Omega)}+\|g\|_{L^{1}(\partial \Omega)}\right) .
\end{gathered}
$$

Proof of Lemma 3.2, By taking $v=T_{k}\left(u_{n}\right)$ in the weak formulation (3.3), we obtain

$$
\begin{aligned}
\int_{\Omega} T_{n}\left(b\left(u_{n}\right)\right) T_{k}\left(u_{n}\right) d x & +\int_{\Omega} a\left(x, u_{n}, \nabla u_{n}\right) \cdot \nabla T_{k}\left(u_{n}\right) d x+\int_{\partial \Omega} \lambda T_{n}\left(u_{n}\right) T_{k}\left(u_{n}\right) d \sigma \\
& +\varepsilon \int_{\Omega}\left[\left|u_{n}\right|^{p_{+}-2} u_{n} T_{k}\left(u_{n}\right)+\left|\nabla u_{n}\right|^{p_{+}-2} \nabla u_{n} \nabla T_{k}\left(u_{n}\right)\right] d x \\
& =\int_{\Omega} f_{n} T_{k}\left(u_{n}\right) d x+\int_{\partial \Omega} g_{n} T_{k}\left(u_{n}\right) d \sigma .
\end{aligned}
$$

Since all the terms of the left hand side of (3.7) are nonnegative, we deduce that

$$
\int_{\Omega} a\left(x, u_{n}, \nabla u_{n}\right) \cdot \nabla T_{k}\left(u_{n}\right) d x \leq \int_{\Omega} f_{n} T_{k}\left(u_{n}\right) d x+\int_{\partial \Omega} g_{n} T_{k}\left(u_{n}\right) d \sigma .
$$


By using $\left(A_{6}\right)$ and (3.8), we get

$$
\begin{aligned}
\int_{\Omega}\left|\nabla T_{k}\left(u_{n}\right)\right|^{\pi_{n}(.)} d x & \leq C_{2}\left(\int_{\Omega} f_{n} T_{k}\left(u_{n}\right) d x+\int_{\partial \Omega} g_{n} T_{k}\left(u_{n}\right) d \sigma\right) \\
& \leq C_{2} k\left(\|f\|_{L^{1}(\Omega)}+\|g\|_{L^{1}(\partial \Omega)}\right) .
\end{aligned}
$$

From (3.7), we deduce that

$$
\begin{aligned}
\int_{\Omega} T_{n}\left(b\left(u_{n}\right)\right) T_{k}\left(u_{n}\right) d x & \leq \int_{\Omega} f_{n} T_{k}\left(u_{n}\right) d x+\int_{\partial \Omega} g_{n} T_{k}\left(u_{n}\right) d \sigma \\
& \leq k\left(\|f\|_{L^{1}(\Omega)}+\|g\|_{L^{1}(\partial \Omega)}\right)
\end{aligned}
$$

and

$$
\begin{aligned}
\lambda \int_{\partial \Omega} T_{n}\left(u_{n}\right) T_{k}\left(u_{n}\right) d x & \leq \int_{\Omega} f_{n} T_{k}\left(u_{n}\right) d x+\int_{\partial \Omega} g_{n} T_{k}\left(u_{n}\right) d \sigma \\
& \leq k\left(\|f\|_{L^{1}(\Omega)}+\|g\|_{L^{1}(\partial \Omega)}\right) .
\end{aligned}
$$

Dividing (3.9) and (3.10) by $k$ and letting $k$ goes to 0 , we obtain

$$
\int_{\Omega} T_{n}\left(b\left(u_{n}\right)\right) \operatorname{sign}_{0}\left(u_{n}\right) d x \leq\|f\|_{L^{1}(\Omega)}+\|g\|_{L^{1}(\partial \Omega)}
$$

and

$$
\lambda \int_{\partial \Omega} T_{n}\left(u_{n}\right) \operatorname{sign}_{0}\left(u_{n}\right) d x \leq\|f\|_{L^{1}(\Omega)}+\|g\|_{L^{1}(\partial \Omega)} .
$$

Hence,

$$
\int_{\Omega}\left|T_{n}\left(b\left(u_{n}\right)\right)\right| d x \leq\|f\|_{L^{1}(\Omega)}+\|g\|_{L^{1}(\partial \Omega)}
$$

and

$$
\int_{\partial \Omega}\left|T_{n}\left(u_{n}\right)\right| d x \leq \frac{1}{\lambda}\left(\|f\|_{L^{1}(\Omega)}+\|g\|_{L^{1}(\partial \Omega)}\right) .
$$

Lemma 3.3. Assume that $\left(A_{3}\right)-\left(A_{6}\right)$ hold. If $u_{n}$ is a weak solution of the problem (3.2), $f_{n} \in$ $L^{\infty}(\Omega)$ and $g_{n} \in L^{\infty}(\partial \Omega)$, then for all $k>0$

$$
\int_{\Omega}\left|\nabla T_{k}\left(u_{n}\right)\right|^{p_{-}} d x \leq C\left(\|f\|_{L^{1}(\Omega)},\|g\|_{L^{1}(\partial \Omega)}, \text { meas }(\Omega)\right)(k+1)
$$

and

$$
\int_{\partial \Omega}\left|T_{k}\left(u_{n}\right)\right| d \sigma \leq \frac{1}{\lambda}\left(\|f\|_{L^{1}(\Omega)}+\|g\|_{L^{1}(\partial \Omega)}\right),
$$

for all $n \geq k>0$. 
Proof of Lemma 3.3 Firstly, we prove (3.11). We know that

$$
\int_{\Omega}\left|\nabla T_{k}\left(u_{n}\right)\right|^{\pi_{n}(\cdot)} d x \leq C_{2} k\left(\|f\|_{L^{1}(\Omega)}+\|g\|_{L^{1}(\partial \Omega)}\right) .
$$

Let us note that

$$
\begin{aligned}
\int_{\Omega}\left|\nabla T_{k}\left(u_{n}\right)\right|^{p_{-}} d x & =\int_{\left\{\left|\nabla T_{k}\left(u_{n}\right)\right|>1\right\}}\left|\nabla T_{k}\left(u_{n}\right)\right|^{p_{-}} d x+\int_{\left\{\left|\nabla T_{k}\left(u_{n}\right)\right| \leq 1\right\}}\left|\nabla T_{k}\left(u_{n}\right)\right|^{p_{-}} d x \\
& \leq \int_{\left\{\left|\nabla T_{k}\left(u_{n}\right)\right|>1\right\}}\left|\nabla T_{k}\left(u_{n}\right)\right|^{p_{-}} d x+\text { meas }(\Omega) \\
& \leq \int_{\Omega}\left|\nabla T_{k}\left(u_{n}\right)\right|^{\pi_{n}(\cdot)} d x+\operatorname{meas}(\Omega)
\end{aligned}
$$

By using (3.13) and (3.14), we get

$$
\begin{aligned}
\int_{\Omega}\left|\nabla T_{k}\left(u_{n}\right)\right|^{p_{-}} d x & \leq \max \left(C_{2}\left(\|f\|_{L^{1}(\Omega)}+\|g\|_{L^{1}(\partial \Omega)}\right), \text { meas }(\Omega)\right)(k+1) \\
& :=C\left(\|f\|_{L^{1}(\Omega)},\|g\|_{L^{1}(\partial \Omega)}, \text { meas }(\Omega)\right)(k+1) .
\end{aligned}
$$

Now, from the formula (3.6), we obtain $\left\|T_{n}\left(u_{n}\right)\right\|_{L^{1}(\partial \Omega)} \leq \frac{1}{\lambda}\left(\|f\|_{L^{1}(\Omega)}+\|g\|_{L^{1}(\partial \Omega)}\right)$ and as $\left|T_{k}\left(u_{n}\right)\right| \leq\left|T_{n}\left(u_{n}\right)\right|$ for all $n \geq k>0$, one deduces that

$$
\int_{\partial \Omega}\left|T_{k}\left(u_{n}\right)\right| d \sigma \leq \frac{1}{\lambda}\left(\|f\|_{L^{1}(\Omega)}+\|g\|_{L^{1}(\partial \Omega)}\right) .
$$

Lemma 3.4. For any $k>0$, we have

$$
\left\|T_{k}\left(u_{n}\right)\right\|_{W^{1, \pi_{n}(\cdot)(\Omega)}} \leq 1+C\left(k, f, g, p_{-}, p_{+}, \operatorname{meas}(\Omega)\right)
$$

and for all $k \geq 1$,

$$
\operatorname{meas}\left(\left\{\left|u_{n}\right|>k\right\}\right) \leq \frac{C}{\min (b(k),|b(-k)|)}
$$

Proof of Lemma 3.4, By using (3.4), we have

$$
\int_{\Omega}\left|\nabla T_{k}\left(u_{n}\right)\right|^{\pi_{n}(\cdot)} d x \leq C_{2} k\left(\|f\|_{L^{1}(\Omega)}+\|g\|_{L^{1}(\partial \Omega)}\right) .
$$

We also have

$$
\int_{\Omega}\left|T_{k}\left(u_{n}\right)\right|^{\pi_{n}(.)} d x=\int_{\left\{\left|u_{n}\right| \leq k\right\}}\left|T_{k}\left(u_{n}\right)\right|^{\pi_{n}(.)} d x+\int_{\left\{\left|u_{n}\right|>k\right\}}\left|T_{k}\left(u_{n}\right)\right|^{\pi_{n}(.)} d x .
$$

Furthermore,

$$
\begin{aligned}
\int_{\left\{\left|u_{n}\right|>k\right\}}\left|T_{k}\left(u_{n}\right)\right|^{\pi_{n}(.)} d x & =\int_{\left\{\left|u_{n}\right|>k\right\}} k^{\pi_{n}(\cdot)} d x \\
& \leq\left\{\begin{array}{lll}
k^{p_{+}} \operatorname{meas}(\Omega) & \text { if } \quad k \geq 1 \\
\operatorname{meas}(\Omega) & \text { if } & k<1
\end{array}\right.
\end{aligned}
$$


and

$$
\begin{aligned}
\int_{\left\{\left|u_{n}\right| \leq k\right\}}\left|T_{k}\left(u_{n}\right)\right|^{\pi_{n}(.)} d x & \leq \int_{\left\{\left|u_{n}\right| \leq k\right\}} k^{\pi_{n}(.)} d x \\
& \leq\left\{\begin{array}{lll}
k^{p_{+}} \operatorname{meas}(\Omega) & \text { if } \quad k \geq 1 \\
\operatorname{meas}(\Omega) & \text { if } \quad k<1 .
\end{array}\right.
\end{aligned}
$$

This allow us to write

$$
\int_{\Omega}\left|T_{k}\left(u_{n}\right)\right|^{\pi_{n}(.)} d x \leq 2\left(1+k^{p_{+}}\right) \operatorname{meas}(\Omega) .
$$

Hence, adding (3.16) and (3.17) one gets

$$
\rho_{1, \pi_{n}(.)}\left(T_{k}\left(u_{n}\right)\right) \leq C_{2} k\left(\|f\|_{L^{1}(\Omega)}+\|g\|_{L^{1}(\partial \Omega)}\right)+2\left(1+k^{p_{+}}\right) \operatorname{meas}(\Omega) .
$$

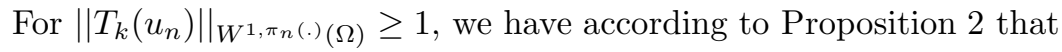

$$
\left\|T_{k}\left(u_{n}\right)\right\|_{W^{1, \pi_{n}(.)(\Omega)}}^{p_{-}} \leq \rho_{1, \pi_{n}(.)}\left(T_{k}\left(u_{n}\right)\right) \leq\left[C_{2} k\left(\|f\|_{L^{1}(\Omega)}+\|g\|_{L^{1}(\partial \Omega)}\right)+2\left(1+k^{p_{+}}\right) \operatorname{meas}(\Omega)\right],
$$

which implies that

$$
\begin{aligned}
\left\|T_{k}\left(u_{n}\right)\right\|_{W^{1, \pi_{n}(.)}(\Omega)} & \leq\left[C_{2} k\left(\|f\|_{L^{1}(\Omega)}+\|g\|_{L^{1}(\partial \Omega)}\right)+2\left(1+k^{p_{+}}\right) \operatorname{meas}(\Omega)\right]^{\frac{1}{p_{-}}} \\
& :=C\left(k, f, g, p_{+}, p_{-}, \text {meas }(\Omega)\right) .
\end{aligned}
$$

Thus,

$$
\left\|T_{k}\left(u_{n}\right)\right\|_{W^{1, \pi_{n}(.)(\Omega)}}<1+C\left(k, f, g, p_{+}, p_{-}, \operatorname{meas}(\Omega)\right) .
$$

Moreover, from (3.5), we have

$$
\int_{\partial \Omega}\left|T_{n}\left(b\left(u_{n}\right)\right)\right| d x \leq\|f\|_{L^{1}(\Omega)}+\|g\|_{L^{1}(\partial \Omega)} .
$$

We deduce that the sequence $\left(T_{n}\left(b\left(u_{n}\right)\right)\right)_{n \in \mathbb{N}^{*}}$ is uniformly bounded in $L^{1}(\Omega)$. Thus, $\left(b\left(u_{n}\right)\right)_{n \in \mathbb{N}^{*}}$ is uniformly bounded in $L^{1}(\Omega)$. So, there exists a positive constant $C$ such that

$$
\int_{\Omega}\left|b\left(u_{n}\right)\right| d x \leq C
$$

Furthermore, for all $k \geq 1$, we have

$$
\int_{\left\{\left|u_{n}\right|>k\right\}}\left|b\left(u_{n}\right)\right| d x \leq \int_{\Omega}\left|b\left(u_{n}\right)\right| d x \leq C .
$$

As $b$ is continuous, nondecreasing and surjective, we infer

$$
\int_{\left\{\left|u_{n}\right|>k\right\}} \min (b(k),|b(-k)|) d x \leq \int_{\left\{\left|u_{n}\right|>k\right\}}\left|b\left(u_{n}\right)\right| d x \leq C .
$$

Therefore,

$$
\operatorname{meas}\left(\left\{\left|u_{n}\right|>k\right\}\right) \leq \frac{C}{\min (b(k),|b(-k)|)}, \quad \forall k \geq 1
$$


Then, the proof of Lemma 3.4 is complete.

From the Lemma 3.4 we deduce that for any $k>0$, the sequence $\left(T_{k}\left(u_{n}\right)\right)_{n \in \mathbb{N}}$ is uniformly bounded in $W^{1, \pi_{n}(\cdot)}(\Omega)$ and also in $W^{1, p_{-}}(\Omega)$.

Then, up to a subsequence still denoted $T_{k}\left(u_{n}\right)$, we can assume that for any $k>0, T_{k}\left(u_{n}\right)$ weakly converges to $s_{k}$ in $W^{1, p_{-}}(\Omega)$ and also $T_{k}\left(u_{n}\right)$ strongly converges to $s_{k}$ in $L^{p_{-}}(\Omega)$.

By using the above a priori estimates, we obtain the following convergence results .

\section{Step 2: The convergence results}

The proof of the following proposition use the Lemma 3.4 .

Proposition 6. Assume that $\left(A_{3}\right)-\left(A_{6}\right)$ hold and let $u_{n}$ be a weak solution of the problem (3.2), then the sequence $\left(u_{n}\right)_{n \in \mathbb{N}}$ is Cauchy in measure.

In particular, there exists a measurable function $u$ and a subsequence still denoted $u_{n}$ such that $u_{n} \rightarrow u$ in measure, as $n \rightarrow+\infty$.

As $\left(u_{n}\right)_{n \in \mathbb{N}}$ is a Cauchy sequence in measure, so (up to a subsequence) it converges almost everywhere to some measurable function $u$.

As for any $k>0, T_{k}$ is continuous; then $T_{k}\left(u_{n}\right) \rightarrow T_{k}(u)$ a.e. $x \in \Omega$, so $s_{k}=T_{k}(u)$.

Therefore,

$$
T_{k}\left(u_{n}\right) \rightarrow T_{k}(u) \text { in } W^{1, p_{-}}(\Omega)
$$

and by compact embedding Theorem, we have

$T_{k}\left(u_{n}\right) \rightarrow T_{k}(u)$ in $L^{p_{-}}(\Omega)$ (respectively in $L^{p_{-}}(\partial \Omega)$ ) and a.e. in $\Omega$ (respectively a.e. on $\partial \Omega$ ).

Lemma 3.5. $u_{n}$ converges a.e. on $\partial \Omega$ to some function $v$.

\section{Proof of Lemma 3.5}

Since $T_{k}\left(u_{n}\right) \rightarrow T_{k}(u)$ in $W^{1, p_{-}}(\Omega)$ and $W^{1, p_{-}}(\Omega) \hookrightarrow L^{p_{-}}(\partial \Omega)$ (compact embedding), then $T_{k}\left(u_{n}\right) \rightarrow T_{k}(u)$ in $L^{p_{-}}(\partial \Omega)$ and a.e. on $\partial \Omega$. Therefore, $T_{k}\left(u_{n}\right) \rightarrow T_{k}(u)$ in $L^{1}(\partial \Omega)$ and a.e. in $\partial \Omega$. We deduce that there exists $E \subset \partial \Omega$ such that $T_{k}\left(u_{n}\right) \rightarrow T_{k}(u)$ on $\partial \Omega \backslash E$ with $\mu(E)=0$, where $\mu$ is area measure on $\partial \Omega$.

For every $k>0$, let $E_{k}=\left\{x \in \partial \Omega\right.$ such that $\left.\left|T_{k}(u)\right|<k\right\}$ and $F=\partial \Omega \backslash \bigcup_{k>0} E_{k}$. By using Fatou's Lemma, we have

$$
\begin{aligned}
\int_{\partial \Omega}\left|T_{k}(u)\right| d \sigma & \leq \liminf _{n \rightarrow+\infty} \int_{\partial \Omega}\left|T_{k}\left(u_{n}\right)\right| d \sigma \\
& \leq \frac{\|f\|_{L^{1}(\Omega)}+\|g\|_{L^{1}(\partial \Omega)}}{\lambda} .
\end{aligned}
$$


Now, we use (3.18) to get

$$
\begin{aligned}
\mu(F)=\frac{1}{k} \int_{F}\left|T_{k}(u)\right| d \sigma & \leq \frac{1}{k} \int_{\partial \Omega}\left|T_{k}(u)\right| d \sigma \\
& \leq \frac{\|f\|_{L^{1}(\Omega)}+\|g\|_{L^{1}(\partial \Omega)}}{k \lambda} .
\end{aligned}
$$

We obtain $\mu(F)=0$, as $k$ goes to $\infty$. Let's now define on $\partial \Omega$ the function $v$ by

$$
v(x)=T_{k}(u(x)), \quad x \in E_{k} .
$$

We take $x \in \partial \Omega \backslash(E \cup F)$, then there exists $k>0$ such that $x \in E_{k}$ and we have

$$
u_{n}(x)-v(x)=\left(u_{n}(x)-T_{k}\left(u_{n}(x)\right)\right)+\left(T_{k}\left(u_{n}(x)\right)-T_{k}(u(x))\right)
$$

Since $x \in E_{k}$, we have $\left|T_{k}(u(x))\right|<k$ and so $\left|T_{k}\left(u_{n}(x)\right)\right|<k$, from which we deduce that $\left|u_{n}(x)\right|<k$. Therefore,

$$
u_{n}(x)-v(x)=T_{k}\left(u_{n}(x)\right)-T_{k}(u(x)) \rightarrow 0, \text { as } n \rightarrow+\infty .
$$

This means that $u_{n}$ converges to $v$ a.e. on $\partial \Omega$, but for all $x \in E_{k}, T_{k}(u(x))=u(x)$. Thus, $v=u$ a.e. on $\partial \Omega$. Therefore,

$$
u_{n} \rightarrow u \text { a.e. on } \partial \Omega \text {. }
$$

The following assertions are based on the Young measure and nonlinear weak $-{ }^{*}$ convergence results (see [7, 19, 13]).

\section{Step 3: The convergence in term of Young measure}

\section{Assertion 1}

The sequence $\left(\nabla T_{k}\left(u_{n}\right)\right)_{n \in \mathbb{N}}$ converges to a Young measure $\nu_{x}^{k}(\lambda)$ on $\mathbb{R}^{N}$ in the sense of the nonlinear weak-* convergence and

$$
\nabla T_{k}(u)=\int_{\mathbb{R}^{N}} \lambda d \nu_{x}^{k}(\lambda)
$$

Proof. Using Lemma 3.3. $\nabla T_{k}\left(u_{n}\right)$ is uniformly bounded in $L^{p_{-}}(\Omega)$, so, equi-integrable on $\Omega$. Moreover, $\nabla T_{k}\left(u_{n}\right)$ weakly converges to $\nabla T_{k}(u)$ in $L^{p_{-}}(\Omega)$. Therefore, using the representation of weakly convergence sequences in $L^{1}(\Omega)$ in terms of Young measures (see Theorem 2.1 and formula (2.5)), we can write

$$
\nabla T_{k}(u)=\int_{\mathbb{R}^{N}} \lambda d \nu_{x}^{k}(\lambda)
$$

Assertion 2. $|\lambda|^{\pi(.)}$ is integrable with respect to the measure $\nu_{x}^{k}(\lambda) d x$ on $\mathbb{R}^{N} \times \Omega$, moreover, $T_{k}(u) \in W^{1, \pi(\cdot)}(\Omega)$.

Proof. We know that $p\left(., u_{n}().\right) \rightarrow p(., u()$.$) in measure on \Omega$. Now, using Theorem 2.1 (ii), 
(iii) $\left(p\left(., u_{n}(.)\right), \nabla T_{k}\left(u_{n}\right)\right)_{n \in \mathbb{N}}$ converges on $\mathbb{R} \times \mathbb{R}^{N}$ to Young measure $\mu_{x}^{k}=\delta_{\pi(x)} \otimes \nu_{x}^{k}$.

Thus, we can apply the weak convergence properties (2.4) to the Carathodory function $F_{m}\left(x, \lambda_{0}, \lambda\right) \in \Omega \times\left(\mathbb{R} \times \mathbb{R}^{N}\right) \mapsto\left|h_{m}(\lambda)\right|^{\lambda_{0}}$ with $m \in \mathbb{N}$, where $h_{m}$ is defined in the preliminaries. Then, we obtain

$$
\begin{aligned}
\int_{\Omega \times \mathbb{R}^{N}}\left|h_{m}(\lambda)\right|^{\pi(x)} d \nu_{x}^{k}(\lambda) d x & =\int_{\Omega \times\left(\mathbb{R} \times \mathbb{R}^{N}\right)}\left|h_{m}(\lambda)\right|^{\lambda_{0}} d \mu_{x}^{k}\left(\lambda_{0}, \lambda\right) d x \\
& =\int_{\Omega} \int_{\mathbb{R} \times \mathbb{R}^{N}} F_{m}\left(x, \lambda_{0}, \lambda\right) d \mu_{x}^{k}\left(\lambda_{0}, \lambda\right) d x \\
& =\lim _{n \rightarrow+\infty} \int_{\Omega} F_{m}\left(x, p\left(x, u_{n}(x)\right), \nabla T_{k}\left(u_{n}(x)\right) d x\right. \\
& =\lim _{n \rightarrow+\infty} \int_{\Omega}\left|h_{m}\left(\nabla T_{k}\left(u_{n}\right)\right)\right|^{p\left(., u_{n}(.)\right)} d x \\
& \leq \lim _{n \rightarrow+\infty} \int_{\Omega}\left|\nabla T_{k}\left(u_{n}\right)\right|^{p\left(., u_{n}(.)\right)} d x \\
& \left.\leq C_{2} k\left(\|f\|_{L^{1}(\Omega)}+\|g\|_{L^{1}(\partial \Omega)}\right) \quad \text { (using (3.4) }\right) .
\end{aligned}
$$

$h_{m}(\lambda) \rightarrow \lambda$, as $m \rightarrow+\infty$ and $m \mapsto h_{m}(\lambda)$ is increasing. Then, using Lebesgue convergence Theorem, we deduce from last inequality that

$$
\int_{\Omega \times \mathbb{R}^{N}}|\lambda|^{\pi(x)} d \nu_{x}^{k}(\lambda) d x \leq C_{2} k\left(\|f\|_{L^{1}(\Omega)}+\|g\|_{L^{1}(\partial \Omega)}\right) .
$$

Hence, $|\lambda|^{\pi(.)}$ is integrable with respect to the measure $\nu_{x}^{k}(\lambda) d x$ on $\mathbb{R}^{N} \times \Omega$.

From (3.19), the last inequality and Jensen inequality, we get

$$
\int_{\Omega}\left|\nabla T_{k}(u)\right|^{\pi(x)} d x=\int_{\Omega}\left|\int_{\mathbb{R}^{N}} \lambda d \nu_{x}^{k}(\lambda)\right|^{\pi(x)} d x \leq \int_{\Omega \times \mathbb{R}^{N}}|\lambda|^{\pi(x)} d \nu_{x}^{k} d x<\infty .
$$

Thus, $\nabla T_{k}(u) \in L^{\pi(.)}(\Omega)$. Moreover, $\int_{\Omega}\left|T_{k}(u)\right|^{\pi(.)} d x \leq \max \left(k^{p_{+}}, k^{p_{-}}\right)$meas $(\Omega)$. Hence, $T_{k}(u) \in$ $L^{\pi(.)}(\Omega)$ and we conclude that $T_{k}(u) \in W^{1, \pi(\cdot)}(\Omega)$.

\section{Assertion 3.}

i) The sequence $\left(\Phi_{n}^{k}\right)_{n \in \mathbb{N}}$ defined by $\Phi_{n}^{k}:=a\left(x, u_{n}, \nabla T_{k}\left(u_{n}\right)\right)$ is equi-integrable on $\Omega$.

ii) The sequence $\left(\Phi_{n}^{k}\right)_{n \in \mathbb{N}}$ weakly converges to $\Phi^{k}$ in $L^{1}(\Omega)$ and we have

$$
\Phi^{k}(x)=\int_{\mathbb{R}^{N}} a(x, u, \lambda) d \nu_{x}^{k}(\lambda) .
$$

Proof. i) Using the growth assumption $\left(A_{5}\right)$ with variable exponent $p\left(., u_{n}().\right)$ and relation (3.4), we deduce that $\left(\Phi_{n}^{k}\right)$ is bounded in $L^{\pi_{n}^{\prime}(.)}(\Omega)$, so, $L^{\pi_{n}^{\prime}(\cdot)}$ - equi-integrable on $\Omega$.

Moreover, as $\pi_{n}^{\prime}()>$.1 , we obtain

$$
\left|a\left(x, u_{n}, \nabla T_{k}\left(u_{n}\right)\right)\right| \leq 1+\left|a\left(x, u_{n}, \nabla T_{k}\left(u_{n}\right)\right)\right|^{\pi_{n}^{\prime}(.)} .
$$


Thus, for all subset $E \subset \Omega$, we have

$$
\int_{E}\left|a\left(x, u_{n}, \nabla T_{k}\left(u_{n}\right)\right)\right| d x \leq \operatorname{meas}(E)+\int_{E}\left|a\left(x, u_{n}, \nabla T_{k}\left(u_{n}\right)\right)\right|^{\pi_{n}^{\prime}(.)} d x .
$$

Therefore, for meas $(E)$ small enough, $\left(\Phi_{n}^{k}\right)$ is equi-integrable on $\Omega$.

ii) Set $\tilde{\Phi}_{n}^{k}=a\left(x, u(x), \nabla v_{n}\right)$ with $\nabla v_{n}=\nabla T_{k}\left(u_{n}\right) \cdot \chi_{S_{n}}$ where $S_{n}=\left\{x \in \Omega, \quad\left|\pi(x)-\pi_{n}(x)\right|<\frac{1}{2}\right\}$. Applying $\left(A_{5}\right)$ with variable exponent $\pi($.$) on a\left(x, u(x), \nabla v_{n}\right)$, we have for all subset $E \subset \Omega$,

$$
\begin{aligned}
\int_{E}\left|a\left(x, u(x), \nabla v_{n}\right)\right| d x & \leq C \int_{E}\left(1+\mathcal{M}(x)+\left|\nabla v_{n}\right|^{\pi(.)-1}\right) d x \\
& \leq C \int_{E}(1+\mathcal{M}(x)) d x+\int_{E \cap S_{n}}\left|\nabla T_{k}\left(u_{n}\right)\right|^{\pi(.)-1} d x .
\end{aligned}
$$

The first term of the right hand side of the last inequality is small for meas $(E)$ small enough. For $x \in S_{n}, \pi(x)<\pi_{n}(x)+\frac{1}{2}$, thus

$$
\int_{E \cap S_{n}}\left|\nabla T_{k}\left(u_{n}\right)\right|^{\pi(.)-1} d x \leq \int_{E \cap S_{n}}\left(1+\left|\nabla T_{k}\left(u_{n}\right)\right|^{\pi_{n}(.)-\frac{1}{2}}\right) d x
$$

and

$$
\int_{\Omega}\left|\nabla T_{k}\left(u_{n}\right)\right|^{\left(\pi_{n}(.)-\frac{1}{2}\right)\left(2 \pi_{n}(.)\right)^{\prime}} d x=\int_{\Omega}\left|\nabla T_{k}\left(u_{n}\right)\right|^{\pi_{n}(.)} d x<\infty
$$

which is equivalent to saying $\left|\nabla T_{k}\left(u_{n}\right)\right|^{\pi_{n}(.)-\frac{1}{2}} \in L^{\left(2 \pi_{n}(.)\right)^{\prime}}(\Omega)$. Now, using Hlder type inequality,

$$
\begin{aligned}
\int_{E \cap S_{n}}\left|\nabla T_{k}\left(u_{n}\right)\right|^{\pi(.)-1} d x & \leq \int_{E}\left(1+\left|\nabla T_{k}\left(u_{n}\right)\right|^{\pi_{n}(.)-\frac{1}{2}}\right) d x \\
& \leq \operatorname{meas}(E)+2\left\|\nabla T_{k}\left(u_{n}\right)\right\|_{L^{\pi_{n}(.)}(\Omega)}\left\|\chi_{E}\right\|_{L^{2 \pi_{n}(.)(\Omega)}} .
\end{aligned}
$$

According to Proposition 1 ,

$$
\begin{aligned}
\left\|\chi_{E}\right\|_{L^{2 \pi_{n}(.)}(\Omega)} & \leq \max \left\{\left(\rho_{2 \pi_{n}(.)}\left(\chi_{E}\right)\right)^{\frac{1}{2 p_{-}}},\left(\rho_{2 \pi_{n}(.)}\left(\chi_{E}\right)\right)^{\frac{1}{2 p_{+}}}\right\} \\
& =\max \left\{(\operatorname{meas}(E))^{\frac{1}{2 p}-},(\operatorname{meas}(E))^{\frac{1}{2 p_{+}}}\right\} .
\end{aligned}
$$

The right-hand side of (3.21) is uniformly small for meas $(E)$ small, and the equi-integrability of $\tilde{\Phi}_{n}^{k}$ follows. Therefore, (up to a subsequence) $\tilde{\Phi}_{n}^{k}$ weakly converges in $L^{1}(\Omega)$ to $\tilde{\Phi}^{k}$, as $n \rightarrow+\infty$. Now, we prove that $\tilde{\Phi}^{k}=\Phi^{k} ;$ more precisely, we show that $\tilde{\Phi}_{n}^{k}-\Phi_{n}^{k}$ strongly converges in $L^{1}(\Omega)$ to 0 .

Let $\beta>0$, by (3.4), $\int_{\Omega}\left|\nabla T_{k}\left(u_{n}\right)\right|^{\pi_{n}(.)} d x$ is uniformly bounded, which implies that $\int_{\Omega}\left|\nabla T_{k}\left(u_{n}\right)\right| d x$ is finite, since

$$
\int_{\Omega}\left|\nabla T_{k}\left(u_{n}\right)\right| d x \leq \int_{\Omega}\left(1+\left|\nabla T_{k}\left(u_{n}\right)\right|^{\pi_{n}(x)}\right) d x .
$$

By Chebyschev Inequality, we have

$$
\operatorname{meas}\left(\left\{\left|\nabla T_{k}\left(u_{n}\right)\right|>L\right\}\right) \leq \frac{\int_{\Omega}\left|\nabla T_{k}\left(u_{n}\right)\right| d x}{L} .
$$


Therefore, $\sup _{n \in \mathbb{N}}$ meas $\left(\left\{\left|\nabla T_{k}\left(u_{n}\right)\right|>L\right\}\right)$ tends to 0 for $L$ large enough. Since $\tilde{\Phi}_{n}^{k}-\Phi_{n}^{k}$ is equiintegrable, there exists $\delta=\delta(\beta)$ such that for all $A \subset \Omega$, meas $(A)<\delta$ and $\int_{A}\left|\tilde{\Phi}_{n}^{k}-\Phi_{n}^{k}\right| d x<\frac{\beta}{4}$.

Therefore, if we choose $L$ large enough, we get $\frac{\int_{\Omega}\left|\nabla T_{k}\left(u_{n}\right)\right| d x}{L}<\delta$, so meas $\left(\left\{\left|\nabla T_{k}\left(u_{n}\right)\right|>L\right\}\right)<\delta$. Hence,

$$
\int_{\left\{\left|\nabla T_{k}\left(u_{n}\right)\right|>L\right\}}\left|\tilde{\Phi}_{n}^{k}-\Phi_{n}^{k}\right| d x<\frac{\beta}{4} .
$$

By Lemma 2.2, we also have

$$
\operatorname{meas}\left(\left\{x \in \Omega ; \sup _{\lambda \in K}\left|a\left(x, u_{n}(x), \lambda\right)-a(x, u(x), \lambda)\right| \geq \sigma\right\}\right) \rightarrow 0,
$$

as $n \rightarrow+\infty$.

Thus, by the above equi-integrability, for all $\sigma>0$, there exists $n_{0}=n_{0}(\sigma, L) \in \mathbb{N}$ such that for all $n \geq n_{0}$,

$$
\int_{\left\{x \in \Omega ; \sup _{|\lambda| \leq L}\left|a\left(x, u_{n}(x), \lambda\right)-a(x, u(x), \lambda)\right| \geq \sigma\right\}}\left|\tilde{\Phi}_{n}^{k}-\Phi_{n}^{k}\right| d x<\frac{\beta}{4} .
$$

Using the definition of $\Phi_{n}^{k}$ and $\tilde{\Phi}_{n}^{k}$, we have

$$
\Phi_{n}^{k}-\tilde{\Phi}_{n}^{k}=a\left(x, u_{n}(x), \nabla T_{k}\left(u_{n}\right)\right)-a\left(x, u(x), \nabla T_{k}\left(u_{n}\right)\right) \text { on } S_{n} .
$$

Now, we reason on

$$
S_{n, L, \sigma}:=\left\{x \in \Omega ; \sup _{|\lambda| \leq L}\left|a\left(x, u_{n}(x), \lambda\right)-a(x, u(x), \lambda)\right|<\sigma,\left|\nabla T_{k}\left(u_{n}\right)\right| \leq L\right\} .
$$

We get

$$
\begin{aligned}
\int_{S_{n, L, \sigma}}\left|\tilde{\Phi}_{n}^{k}-\Phi_{n}^{k}\right| d x & \leq \int_{S_{n, L, \sigma}|\lambda| \leq L} \sup _{|\lambda|}\left|a\left(x, u_{n}(x), \lambda\right)-a(x, u(x), \lambda)\right| d x \\
& \leq \operatorname{\sigma meas}(\Omega) .
\end{aligned}
$$

We observe that

$$
\int_{S_{n}}\left|\tilde{\Phi}_{n}^{k}-\Phi_{n}^{k}\right| d x=\int_{S_{n} \cap S_{n, L, \sigma}}\left|\tilde{\Phi}_{n}^{k}-\Phi_{n}^{k}\right| d x+\int_{S_{n} \backslash S_{n, L, \sigma}}\left|\tilde{\Phi}_{n}^{k}-\Phi_{n}^{k}\right| d x
$$

and

$$
S_{n} \backslash S_{n, L, \sigma} \subset\left\{x \in \Omega ; \sup _{|\lambda| \leq L}\left|a\left(x, u_{n}(x), \lambda\right)-a(x, u(x), \lambda)\right| \geq \sigma\right\} \cup\left\{\left|\nabla T_{k}\left(u_{n}\right)\right|>L\right\} .
$$

Consequently, by choosing $\sigma=\sigma(\beta)<\frac{\beta}{4 \operatorname{meas}(\Omega)}$, we get

$$
\int_{S_{n}}\left|\tilde{\Phi}_{n}^{k}-\Phi_{n}\right| d x<\frac{\beta}{4}+\frac{\beta}{4}+\frac{\beta}{4}=\frac{3 \beta}{4}
$$


for all $n \geq n_{0}(\sigma, L)$. By Lemma 2.2. we also have meas $\left(\left\{x \in \Omega,\left|\pi(x)-\pi_{n}(x)\right| \geq \frac{1}{2}\right\}\right) \rightarrow 0$ for $n$ large enough; which means that meas $\left(\Omega \backslash S_{n}\right)$ converges to 0 for $n$ large enough. Thus,

$$
\int_{\Omega \backslash S_{n}}\left|\tilde{\Phi}_{n}^{k}-\Phi_{n}^{k}\right| d x=\int_{\Omega \backslash S_{n}}\left|\Phi_{n}^{k}\right| d x \leq \frac{\beta}{4} .
$$

Therefore, for all $\beta>0$ there exists $n_{0}=n_{0}(\beta)$ such that for all $n \geq n_{0}, \int_{\Omega}\left|\tilde{\Phi}_{n}^{k}-\Phi_{n}^{k}\right| d x \leq \beta$. Hence, $\tilde{\Phi}_{n}^{k}-\Phi_{n}^{k}$ strongly converges to 0 in $L^{1}(\Omega)$. We prove that

$$
\Phi^{k}(x)=\int_{\mathbb{R}^{N}} a(x, u(x), \lambda) d \nu_{x}^{k}(\lambda) \quad \text { a.e. } x \in \Omega \text { and } \Phi^{k} \in L^{\pi^{\prime}(.)}(\Omega) .
$$

Notice that

$$
\lim _{n \rightarrow+\infty} \int_{\Omega}\left|\nabla T_{k}\left(u_{n}\right)\right|\left(1-\chi_{S_{n}}\right) d x=\lim _{n \rightarrow+\infty} \int_{\Omega \backslash S_{n}}\left|\nabla T_{k}\left(u_{n}\right)\right| d x=0,
$$

since $\left(\nabla T_{k}\left(u_{n}\right)\right)_{n \in \mathbb{N}}$ is equi-integrable and meas $\left(\Omega \backslash S_{n}\right)$ converges to 0 for $n$ large enough. Therefore, $\left(\nabla T_{k}\left(u_{n}\right)\right)_{n \in \mathbb{N}}$ and $\nabla T_{k}\left(u_{n}\right) \chi_{S_{n}}$ converge to the same Young measure $\nu_{x}^{k}(\lambda)$.

Moreover, by applying Theorem 2.1 i) to the Carathodory function $F\left(x,\left(\lambda_{0}, \lambda\right)\right):=a\left(x, \lambda_{0}, \lambda\right)$, we infer that

$$
\tilde{\Phi}(x)=\Phi(x)=\int_{\mathbb{R}^{N}} a(x, u(x), \lambda) d \nu_{x}^{k}(\lambda) \text { a.e. } x \in \Omega .
$$

Using $\left(A_{5}\right)$, it follows that $|a(x, u(x), \lambda)|^{\pi^{\prime}(.)} \leq C\left(\mathcal{M}(x)+|\lambda|^{\pi(.)}\right)$. Thus, with Jensen Inequality, it follows that

$$
\begin{aligned}
\int_{\Omega}\left|\Phi^{k}(x)\right|^{\pi^{\prime}(.)} d x & =\int_{\Omega}\left|\int_{\mathbb{R}^{N}} a(x, u(x), \lambda) d \nu_{x}^{k}(\lambda)\right|^{\pi^{\prime}(.)} d x \\
& \leq \int_{\Omega \times \mathbb{R}^{N}}|a(x, u(x), \lambda)|^{\pi^{\prime}(.)} d \nu_{x}^{k}(\lambda) d x \\
& \leq C \int_{\Omega \times \mathbb{R}^{N}}\left(\mathcal{M}(x)+|\lambda|^{\pi(.)}\right) d \nu_{x}^{k}(\lambda) d x<\infty .
\end{aligned}
$$

Hence, $\Phi^{k} \in L^{\pi^{\prime}(\cdot)}(\Omega)$.

\section{Assertion 4}

(a) For all $k^{\prime}>k>0$, we have $\Phi^{k}=\Phi^{k^{\prime}} \chi_{\{|u|<k\}}$.

(b) For all $k>0$,

$$
\int_{\Omega} \Phi^{k} \cdot \nabla T_{k}(u) d x \geq \int_{\Omega \times \mathbb{R}^{N}} a(x, u(x), \lambda) \cdot \lambda d \nu_{x}^{k}(\lambda) d x .
$$

(c) The div-curl inequality holds:

$$
\int_{\Omega \times \mathbb{R}^{N}}\left(a(x, u(x), \lambda)-a\left(x, u(x), \nabla T_{k}(u(x))\right)\left(\lambda-\nabla T_{k}(u(x))\right) d \nu_{x}^{k}(\lambda) d x \leq 0 .\right.
$$


(d) For all $k>0$,

$$
\Phi^{k}=a\left(x, u(x), \nabla T_{k}(u)\right) \text { for a.e. } x \in \Omega
$$

and $\nabla T_{k}\left(u_{n}\right)$ converges to $\nabla T_{k}(u)$ in measure on $\Omega$, as $n \rightarrow+\infty$.

\section{Proof.}

(a) Let $k^{\prime}>k>0$ and $g_{n}^{k}:=a\left(x, u_{n}, \nabla T_{k^{\prime}}\left(u_{n}\right)\right) \chi_{[|u|<k]}$. By Assertion 3-ii), $\left(g_{n}^{k}\right)_{n \in \mathbb{N}}$ weakly converges to $\Phi^{k^{\prime}} \chi_{[|u|<k]}$ in $L^{1}(\Omega)$. If we prove that $\left(g_{n}^{k}\right)_{n \in \mathbb{N}}$ weakly converges to $\Phi^{k}$ in $L^{1}(\Omega)$, then the wished result will come of the uniqueness of the limit. Let us put

$$
h_{n}^{k}:=a\left(x, u_{n}, \nabla T_{k^{\prime}}\left(u_{n}\right)\right) \chi_{\left[\left|u_{n}\right|<k\right]} .
$$

As $\nabla T_{k}\left(u_{n}\right) \equiv \nabla T_{k^{\prime}}\left(u_{n}\right) \chi_{\left[\left|u_{n}\right|<k\right]}$, for all $k^{\prime}>k>0$, then, we get

$$
h_{n}^{k}:=a\left(x, u_{n}, \nabla T_{k^{\prime}}\left(u_{n}\right)\right) \chi_{\left[\left|u_{n}\right|<k\right]} \equiv a\left(x, u_{n}, \nabla T_{k}\left(u_{n}\right)\right)
$$

so, $\left(h_{n}^{k}\right)_{n \in \mathbb{N}}$ weakly converges to $\Phi^{k}$ in $L^{1}(\Omega)$ by Assertion 3-ii). Set

$$
d_{n}^{k}:=g_{n}^{k}-h_{n}^{k}=a\left(x, u_{n}, \nabla T_{k^{\prime}}\left(u_{n}\right)\right)\left(\chi_{[|u|<k]}-\chi_{\left[\left|u_{n}\right|<k\right]}\right) .
$$

On the one hand, thanks to Assertion 3-i), $\left(d_{n}^{k}\right)_{n \in \mathbb{N}}$ is equi-integrable. On the other hand $d_{n}^{k} \rightarrow 0$ a.e. on $\Omega$. Indeed, $\chi_{\left[\left|u_{n}\right|<k\right]}=\chi_{(-k, k)}\left(u_{n}\right)$ and if $\left|u_{n}\right| \neq k$ a.e. on $\Omega, \chi_{(-k, k)}($.$) is continuous on \mathbb{R}$. In other words $\chi_{(-k, k)}($.$) is continuous on the image of \Omega$ by $u$ a.e. $k>0$. Moreover, $u_{n} \rightarrow u$ a.e. on $\Omega$, then $\chi_{\left[\left|u_{n}\right|<k\right]} \rightarrow \chi_{[|u|<k]}$ a.e. in $\Omega$. Now, using Vitali's Theorem $\left(d_{n}^{k}\right)_{n \in \mathbb{N}}$ strongly converges to 0 in $L^{1}(\Omega)$, so it weakly converges in $L^{1}(\Omega)$. Hence, $\left(g_{n}^{k}\right)_{n \in \mathbb{N}}$ and $\left(h_{n}^{k}\right)_{n \in \mathbb{N}}$ weakly converge to the same limit $\Phi^{k}$ in $L^{1}(\Omega)$.

(b) Let $\mathcal{S}$ be a set of $W^{2, \infty}$ functions $S: \mathbb{R} \rightarrow \mathbb{R}$ such that $S^{\prime}($.$) has a compact support.$

We construct a sequence $\left(S_{M}\right)_{M \in \mathbb{N}} \subset \mathcal{S}$ such that

- $S_{M}^{\prime}$ and $S_{M}^{\prime \prime}$ are uniformly bounded;

- for all $M \in \mathbb{N}, S_{M}^{\prime}=1$ on $[-M+1, M-1], \operatorname{supp} S^{\prime} \subset[-M, M]$;

- the sequence $\left(b(z) S_{M}^{\prime}(z)\right)_{M \in \mathbb{N}}$ is non-decreasing for all $z \in \mathbb{R}$.

For all $\varphi \in C^{\infty}(\bar{\Omega}), v=\varphi S_{M}^{\prime}\left(u_{n}\right)$ is an admissible test function in the weak formulation (3.3). We have

$$
\begin{aligned}
\int_{\Omega} T_{n}\left(b\left(u_{n}\right)\right) S_{M}^{\prime}\left(u_{n}\right) \varphi d x & +\int_{\Omega} S_{M}^{\prime}\left(u_{n}\right) a\left(x, u_{n}, \nabla T_{M}\left(u_{n}\right)\right) \cdot \nabla \varphi d x \\
& +\int_{\Omega} S_{M}^{\prime \prime}\left(u_{n}\right) a\left(x, u_{n}, \nabla T_{M}\left(u_{n}\right)\right) \cdot \nabla T_{M}\left(u_{n}\right) \varphi d x+\int_{\partial \Omega} \lambda T_{n}\left(u_{n}\right) S_{M}^{\prime}\left(u_{n}\right) \varphi d \sigma \\
& +\varepsilon \int_{\Omega}\left[\left|\nabla u_{n}\right|^{p_{+}-2} \nabla u_{n} \nabla\left(\varphi S_{M}^{\prime}\left(u_{n}\right)\right)+\left|u_{n}\right|^{p_{+}-2} u_{n} S_{M}^{\prime}\left(u_{n}\right) \varphi\right] d x \\
& =\int_{\Omega} f_{n} S_{M}^{\prime}\left(u_{n}\right) \varphi d x+\int_{\partial \Omega} g_{n} S_{M}^{\prime}\left(u_{n}\right) \varphi d \sigma .
\end{aligned}
$$


Since $u_{n}$ converges to $u$ a.e. in $\Omega$ and a.e. on $\partial \Omega$, by continuity of $b, S_{M}^{\prime}$ and the compacteness of $\operatorname{supp} S_{M}^{\prime}$, we obtain

$$
\int_{\Omega} T_{n}\left(b\left(u_{n}\right)\right) S_{M}^{\prime}\left(u_{n}\right) \varphi d x \rightarrow \int_{\Omega} b(u) S_{M}^{\prime}(u) \varphi d x, \text { as } n \rightarrow+\infty
$$

and

$$
\lambda \int_{\Omega} T_{n}\left(u_{n}\right) S_{M}^{\prime}\left(u_{n}\right) \varphi d \sigma \rightarrow \lambda \int_{\Omega} u S_{M}^{\prime}(u) \varphi d \sigma, \text { as } n \rightarrow+\infty .
$$

Moreover, we have $\left|f_{n} S_{M}^{\prime}\left(u_{n}\right) \varphi\right| \leq\left\|S_{M}^{\prime}\right\|_{L^{\infty}(\mathbb{R}}|f||\varphi| \in L^{1}(\Omega), f_{n} S_{M}^{\prime}\left(u_{n}\right) \varphi \rightarrow f S_{M}^{\prime}(u) \varphi$ a.e. in $\Omega$. and $\left|g_{n} S_{M}^{\prime}\left(u_{n}\right) \varphi\right| \leq\left\|S_{M}^{\prime}\right\|_{L^{\infty}(\mathbb{R})}|g||\varphi| \in L^{1}(\mathbb{R}), g_{n} S_{M}^{\prime}\left(u_{n}\right) \varphi \rightarrow g S_{M}^{\prime}(u) \varphi$ a.e. on $\partial \Omega$. Thus, by Lebesgue dominated convergence Theorem

$$
\int_{\Omega} f_{n} S_{M}^{\prime}\left(u_{n}\right) \varphi d x \rightarrow \int_{\Omega} f S_{M}^{\prime}(u) \varphi d x, \text { as } n \rightarrow+\infty
$$

and

$$
\int_{\partial \Omega} g_{n} S_{M}^{\prime}\left(u_{n}\right) \varphi d \sigma \rightarrow \int_{\partial \Omega} g S_{M}^{\prime}(u) \varphi d \sigma, \text { as } n \rightarrow+\infty .
$$

Let us prove now, that

$$
\int_{\Omega} S_{M}^{\prime}\left(u_{n}\right) a\left(x, u_{n}, \nabla T_{M}\left(u_{n}\right)\right) . \nabla \varphi d x \rightarrow \int_{\Omega} S_{M}^{\prime}(u) \Phi^{M} \cdot \nabla \varphi d x, \text { as } n \rightarrow+\infty .
$$

For all $L>0$, we have

$$
\begin{aligned}
\int_{\Omega} S_{M}^{\prime}\left(u_{n}\right) a\left(x, u_{n}, \nabla T_{M}\left(u_{n}\right)\right) \cdot \nabla \varphi d x & =\int_{\{|\nabla \varphi| \leq L\}} S_{M}^{\prime}\left(u_{n}\right) \Phi_{n}^{M} \cdot \nabla \varphi d x \\
& +\int_{\{|\nabla \varphi|>L\}} S_{M}^{\prime}\left(u_{n}\right) \Phi_{n}^{M} \cdot \nabla \varphi d x .
\end{aligned}
$$

For the first term of the right-hand side of (3.30), we have

$$
\int_{\{|\nabla \varphi| \leq L\}} S_{M}^{\prime}\left(u_{n}\right) \Phi_{n}^{M} \cdot \nabla \varphi d x \rightarrow \int_{\{|\nabla \varphi| \leq L\}} S_{M}^{\prime}(u) \Phi^{M} \cdot \nabla \varphi d x, \text { as } n \rightarrow+\infty .
$$

Thanks $\Phi_{n}^{M} \rightarrow \Phi^{M}$ in $L^{1}(\Omega)$ and $\nabla \varphi S_{M}^{\prime}\left(u_{n}\right) \chi_{\{|\nabla \varphi| \leq L\}} \rightarrow^{*} \nabla \varphi S_{M}^{\prime}(u) \chi_{\{|\nabla \varphi| \leq L\}}$ in $L^{\infty}(\Omega)$. Furthermore, the second term of the right hand-side of (3.30) converges to zero for $L$ large enough, uniformly in $n$. Indeed, using Hlder type inequality and the fact that $L^{p_{+}}(\Omega) \hookrightarrow L^{\pi_{n}(\cdot)}(\Omega)$, we get

$$
\begin{aligned}
& \left|\int_{\{|\nabla \varphi|>L\}} \Phi_{n}^{M} \nabla \varphi S_{M}^{\prime}\left(u_{n}\right) d x\right| \\
\leq & C|| S_{M}^{\prime}\left\|_{L^{\infty}(\mathbb{R})}|| \Phi_{n}^{M}\right\|_{L^{\pi_{n}^{\prime}(.)}(\Omega)}|| \nabla \varphi \chi_{\{|\nabla \varphi|>L\}} \|_{L^{\pi_{n}(.)}(\Omega)} \\
\leq & C\left(p_{-},\left\|S_{M}^{\prime}\right\|_{L^{\infty}(\mathbb{R})}, \text { meas }(\Omega)\right)\left\|\Phi_{n}^{M}\right\|_{L^{\pi_{n}^{\prime}(.)}(\Omega)}\|\nabla \varphi\|_{L^{p_{+}(\Omega)}} \operatorname{meas}(\{|\nabla \varphi|>L\}) .
\end{aligned}
$$

From $\left(A_{5}\right)$, (3.4) and Proposition 2, we obtain

$$
\left\|\Phi_{M}^{n}\right\|_{L^{\pi_{n}^{\prime}(\cdot)(\Omega)}}<C
$$


Moreover, $\varphi \in C^{\infty}(\bar{\Omega})$ and $C^{\infty}(\bar{\Omega})$ is dense in the space $W^{1, p_{+}}(\Omega)$. Then, by Proposition 2 and the fact that $\lim _{L \rightarrow+\infty} \operatorname{meas}(\{|\nabla \varphi|>L\})=0$, we get

$$
\operatorname{meas}(\{|\nabla \varphi|>L\})\left\|\Phi_{n}^{M}\right\|_{L^{\pi_{n}^{\prime}(\cdot)}(\Omega)}\|\nabla \varphi\|_{L^{p_{+}}(\Omega)} \rightarrow 0, \text { as } L \rightarrow+\infty .
$$

Hence, the second term of the right hand-side of (3.30) converges to zero, as $L$ tends to infinity. Thus, as $n \rightarrow+\infty$ and $L \rightarrow+\infty$ in (3.30), we deduce (3.29).

Let us consider the third term of left hand-side of (3.24), we obtain

$$
\begin{aligned}
\int_{\Omega}\left|S_{M}^{\prime \prime}\left(u_{n}\right)\right| a\left(x, u_{n}, \nabla T_{M}\left(u_{n}\right)\right) \cdot \nabla T_{M}\left(u_{n}\right) \varphi d x & \leq C \int_{\left\{\left|u_{n}\right|<M\right\}}\left|S_{M}^{\prime \prime}\left(u_{n}\right)\right| a\left(x, u_{n}, \nabla T_{M}\left(u_{n}\right)\right) \cdot \nabla T_{M}\left(u_{n}\right) d x \\
& \leq C^{\prime} \int_{\left\{M-1<\left|u_{n}\right|<M\right\}} a\left(x, u_{n}, \nabla T_{M}\left(u_{n}\right)\right) \nabla T_{M}\left(u_{n}\right) d x \\
& +C \int_{\left\{\left|u_{n}\right| \leq M-1\right\}} \underbrace{\mid S_{M}^{\prime \prime}\left(u_{n}\right)}_{=0} \mid a\left(x, u_{n}, \nabla T_{M}\left(u_{n}\right)\right) \cdot \nabla T_{M}\left(u_{n}\right) d x,
\end{aligned}
$$

where $C=C\left(\|\varphi\|_{L^{\infty}(\Omega)}\right), \quad C^{\prime}=C\left(\left\|S_{M}^{\prime \prime}\right\|_{L^{\infty}(\mathbb{R})},\|\varphi\|_{L^{\infty}(\Omega)}\right)$ and $\mid a\left(x, u_{n}, \nabla T_{M}\left(u_{n}\right)\right) . \nabla T_{M}\left(u_{n}\right)$ is finite. Otherwise,

$$
\int_{\left\{M-1<\left|u_{n}\right|<M\right\}} a\left(x, u_{n}, \nabla T_{M}\left(u_{n}\right)\right) \nabla T_{M}\left(u_{n}\right) d x \rightarrow 0, \quad \text { as } M \rightarrow+\infty .
$$

Since, thanks to Lemma3.4, $\lim _{M \rightarrow+\infty} \operatorname{meas}\left(\left\{M-1<\left|u_{n}\right|<M\right\}\right)=0$ and $a\left(x, u_{n}, \nabla T_{M}\left(u_{n}\right)\right) \nabla T_{M}\left(u_{n}\right)$ is equi-integrable.

Finally, using (3.25), (3.26) (3.27), (3.28), (3.29), (3.32) and passing to the limit in (3.24), as $n$ tends to infinty and as $\varepsilon$ goes to 0 , we obtain

$$
\int_{\Omega} b(u) S_{M}^{\prime}(u) \varphi d x+\int_{\Omega} S_{M}^{\prime}(u) \Phi^{M} \cdot \nabla \varphi d x+\lambda \int_{\partial \Omega} u S_{M}^{\prime}(u) \varphi d \sigma=\int_{\Omega} f S_{M}^{\prime}(u) \varphi d x+\int_{\partial \Omega} g S_{M}^{\prime}(u) \varphi d \sigma .
$$

For $k>0$ fixed, $T_{k}(u) \in W^{1, \pi(.)}(\Omega)$ and the exponent $\pi($.$) verify (2.1). Therefore, C^{\infty}(\bar{\Omega})$ is dense in $W^{1, \pi(.)}(\Omega)$, so, we replace $\varphi$ by $T_{k}(u)$. Now, for $M>k$, thanks to (a), we replace $\Phi^{M} \cdot \nabla T_{k}(u)$ by $\Phi^{k} . \nabla T_{k}(u)$ in (3.33).

$S_{M}^{\prime}$ converges a.e. to 1 on $\mathbb{R}$, as $M \rightarrow+\infty$, then using the monotone convergence theorem in the first term of left hand-side of (3.33) and dominated convergence theorem in the other term of (3.33), we get

$$
\int_{\Omega}\left[b(u) T_{k}(u)+\Phi^{k} . \nabla T_{k}(u)\right] d x+\lambda \int_{\partial \Omega} u T_{k}(u) d \sigma=\int_{\Omega} f T_{k}(u) d x+\int_{\partial \Omega} g T_{k}(u) d \sigma .
$$


The relation (3.7) is equivalent to

$$
\begin{aligned}
\int_{\Omega} T_{n}\left(b\left(u_{n}\right)\right) T_{k}\left(u_{n}\right) d x & +\int_{\Omega} a\left(x, u_{n}, \nabla T_{k}\left(u_{n}\right)\right) \cdot \nabla T_{k}\left(u_{n}\right) d x+\int_{\partial \Omega} \lambda T_{n}\left(u_{n}\right) T_{k}\left(u_{n}\right) d \sigma \\
& +\varepsilon \int_{\Omega}\left[\left|u_{n}\right|^{p_{+}-2} u_{n} T_{k}\left(u_{n}\right)+\left|\nabla u_{n}\right|^{p_{+}-2} \nabla u_{n} \nabla T_{k}\left(u_{n}\right)\right] d x \\
& =\int_{\Omega} f_{n} T_{k}\left(u_{n}\right) d x+\int_{\partial \Omega} g_{n} T_{k}\left(u_{n}\right) d \sigma
\end{aligned}
$$

The sequences $\left(T_{n}\left(b\left(u_{n}\right)\right) T_{k}\left(u_{n}\right)\right)_{n \in \mathbb{N}},\left(T_{n}\left(u_{n}\right) T_{k}\left(u_{n}\right)\right)_{n \in \mathbb{N}}$ are nonnegative and converge a.e. in $\Omega$ to $b(u) T_{k}(u)$ and a.e. on $\partial \Omega$ to $u T_{k}(u)$. By Fatou's Lemma, we have

$$
\liminf _{n \rightarrow+\infty} \int_{\Omega} T_{n}\left(b\left(u_{n}\right)\right) T_{k}\left(u_{n}\right) d x \geq \int_{\Omega} b(u) T_{k}(u) d x
$$

and

$$
\lambda \liminf _{n \rightarrow+\infty} \int_{\partial \Omega} T_{n}\left(u_{n}\right) T_{k}\left(u_{n}\right) d x \geq \lambda \int_{\partial \Omega} u T_{k}(u) d \sigma .
$$

Now, we consider the right hand side of (3.35). We have

$\left|f_{n} T_{k}\left(u_{n}\right)\right| \leq k|f| \in L^{1}(\Omega), f_{n} T_{k}\left(u_{n}\right) \rightarrow f T_{k}(u)$ a.e. in $\Omega$ and $\left|g_{n} T_{k}\left(u_{n}\right)\right| \leq k|g| \in L^{1}(\partial \Omega)$, $g_{n} T_{k}\left(u_{n}\right) \rightarrow g T_{k}(u)$ a.e. on $\partial \Omega$. Thus, by Lebesgue dominated convergence Theorem

$$
\int_{\Omega} f_{n} T_{k}\left(u_{n}\right) d x \rightarrow \int_{\Omega} f T_{k}(u) d x, \text { as } n \rightarrow+\infty
$$

and

$$
\int_{\partial \Omega} g_{n} T_{k}\left(u_{n}\right) d \sigma \rightarrow \int_{\partial \Omega} g T_{k}(u) d \sigma, \text { as } n \rightarrow+\infty .
$$

Combining (3.36), (3.37), (3.38), (3.39) and using (3.35), we get

$$
\begin{aligned}
& \liminf _{n \rightarrow+\infty}\left(\int_{\Omega} f_{n} T_{k}\left(u_{n}\right) d x+\int_{\partial \Omega} g_{n} T_{k}\left(u_{n}\right) d \sigma\right)-\left(\int_{\Omega} b(u) T_{k}(u) d x+\lambda \int_{\partial \Omega} u T_{k}(u) d \sigma\right) \\
\geq & \liminf _{n \rightarrow+\infty}\left(\int_{\Omega} f_{n} T_{k}\left(u_{n}\right) d x+\int_{\partial \Omega} g_{n} T_{k}\left(u_{n}\right) d \sigma-\int_{\Omega} T_{n}\left(b\left(u_{n}\right)\right) T_{k}\left(u_{n}\right) d x-\lambda \int_{\partial \Omega} T_{n}\left(u_{n}\right) T_{k}\left(u_{n}\right) d \sigma\right),
\end{aligned}
$$

which is equivalent to

$$
\begin{aligned}
& \int_{\Omega} f T_{k}(u) d x+\int_{\partial \Omega} g T_{k}(u) d \sigma-\left(\int_{\Omega} b(u) T_{k}(u) d x+\lambda \int_{\partial \Omega} u T_{k}(u) d \sigma\right) \\
\geq & \liminf _{n \rightarrow+\infty} \int_{\Omega} a\left(x, u_{n}, \nabla T_{k}\left(u_{n}\right)\right) \nabla T_{k}\left(u_{n}\right) d x+\varepsilon \int_{\Omega}\left[\left|u_{n}\right|^{p_{+}-2} u_{n} T_{k}\left(u_{n}\right)+\left|\nabla u_{n}\right|^{p_{+}-2} \nabla u_{n} \nabla T_{k}\left(u_{n}\right)\right] d x \\
\geq & \liminf _{n \rightarrow+\infty} \int_{\Omega} a\left(x, u_{n}, \nabla T_{k}\left(u_{n}\right) \nabla T_{k}\left(u_{n}\right) d x .\right.
\end{aligned}
$$

Thus, by using the relation (3.34), we obtain

$$
\int_{\Omega} \Phi^{k} \nabla T_{k}(u) d x \geq \liminf _{n \rightarrow+\infty} \int_{\Omega} a\left(x, u_{n}, \nabla T_{k}\left(u_{n}\right)\right) \nabla T_{k}\left(u_{n}\right) d x .
$$


(c) From [1-Lemma 2.1, $m \mapsto a\left(x, u_{n}, h_{m}\left(\nabla T_{k}\left(u_{n}\right)\right)\right) \cdot h_{m}\left(\nabla T_{k}\left(u_{n}\right)\right)$ is increasing and converges to $a\left(x, u_{n}, \nabla T_{k}\left(u_{n}\right)\right) . \nabla T_{k}\left(u_{n}\right)$ for $m$ large enough. Thus, we deduce that

$$
a\left(x, u_{n}, h_{m}\left(\nabla T_{k}\left(u_{n}\right)\right)\right) \cdot h_{m}\left(\nabla T_{k}\left(u_{n}\right)\right) \leq a\left(x, u_{n}, \nabla T_{k}\left(u_{n}\right)\right) \cdot \nabla T_{k}\left(u_{n}\right)=\Phi_{n}^{k} . \nabla T_{k}\left(u_{n}\right) .
$$

Therefore, using (b) and Theorem 2.1, we get

$$
\begin{aligned}
\int_{\Omega} \Phi^{k} \cdot \nabla T_{k}(u) d x & \geq \liminf _{n \rightarrow+\infty} \int_{\Omega} \Phi_{n}^{k} \cdot \nabla T_{k}\left(u_{n}\right) d x \\
& \geq \lim _{n \rightarrow+\infty} \int_{\Omega} a\left(x, u_{n}, h_{m}\left(\nabla T_{k}\left(u_{n}\right)\right)\right) \cdot h_{m}\left(\nabla T_{k}\left(u_{n}\right)\right) d x \\
& =\int_{\Omega \times \mathbb{R}^{N}} a\left(x, u, h_{m}(\lambda)\right) \cdot h_{m}(\lambda) d \nu_{x}^{k}(\lambda) d x
\end{aligned}
$$

Using Lebesgue convergence Theorem in (3.41), we get for $m$ large enough

$$
\int_{\Omega} \Phi^{k} \cdot \nabla T_{k}(u) d x \geq \int_{\Omega \times \mathbb{R}^{N}} a(x, u, \lambda) \cdot \lambda d \nu_{x}^{k}(\lambda) d x .
$$

We have

$$
\begin{aligned}
& \int_{\Omega \times \mathbb{R}^{N}}\left(a(x, u(x), \lambda)-a\left(x, u(x), \nabla T_{k}(u(x))\right)\left(\lambda-\nabla T_{k}(u(x))\right) d \nu_{x}^{k}(\lambda) d x\right. \\
= & \int_{\Omega \times \mathbb{R}^{N}} a(x, u(x), \lambda) \cdot \lambda d \nu_{x}^{k}(\lambda) d x-\int_{\Omega \times \mathbb{R}^{N}} a(x, u(x), \lambda) \cdot \nabla T_{k}(u(x)) d \nu_{x}^{k}(\lambda) d x \\
- & \int_{\Omega \times \mathbb{R}^{N}} a\left(x, u(x), \nabla T_{k}(u(x))\right) \cdot \lambda d \nu_{x}^{k}(\lambda) d x+\int_{\Omega \times \mathbb{R}^{N}} a\left(x, u(x), \nabla T_{k}(u(x))\right) \cdot \nabla T_{k}(u(x)) d \nu_{x}^{k}(\lambda) d x \\
= & \int_{\Omega \times \mathbb{R}^{N}} a(x, u(x), \lambda) \cdot \lambda d \nu_{x}^{k}(\lambda) d x-\int_{\Omega}\left(\int_{\mathbb{R}^{N}} a(x, u(x), \lambda) d \nu_{x}^{k}(\lambda)\right) \nabla T_{k}(u(x)) d x \\
- & \int_{\Omega} a\left(x, u(x), \nabla T_{k}(u(x))\right) \cdot\left(\int_{\mathbb{R}^{N}} \lambda d \nu_{x}^{k}\right) d x+\int_{\Omega} a\left(x, u(x), \nabla T_{k}(u(x))\right) \cdot \nabla T_{k}(u(x))\left(\int_{\mathbb{R}^{N}} d \nu_{x}^{k}\right) d x \\
= & \int_{\Omega \times \mathbb{R}^{N}} a(x, u(x), \lambda) \cdot \lambda d \nu_{x}^{k}(\lambda) d x-\int_{\Omega} \Phi^{k} \cdot \nabla T_{k}(u(x)) d x \leq 0 .
\end{aligned}
$$

We pass from the first equality to the second equality by using Fubini-Tonelli Theorem and from the second inequality to the third one by using (3.19), (3.20) and the fact that $\nu_{x}$ is probability measures on $\mathbb{R}^{N}$. Finally (3.42) give us the desired inequality.

(d) Using (3.23) and the strict monotonicity assumption $\left(A_{4}\right)$, we deduce that

$$
\left(a(x, u(x), \lambda)-a\left(x, u(x), \nabla T_{k}(u(x))\right)\left(\lambda-\nabla T_{k}(u(x))\right)=0 \text { a.e. } x \in \Omega, \quad \lambda \in \mathbb{R}^{N} .\right.
$$

Thus, $\lambda=\nabla T_{k}(u(x))$ a.e. $x \in \Omega$ with respect to the measure $\nu_{x}^{k}$ on $\mathbb{R}^{N}$. Therefore, the measure $\nu_{x}^{k}$ reduces to the Dirac measure $\delta_{\nabla T_{k}(u(x))}$. Using (3.20), we obtain

$$
\Phi^{k}=\int_{\mathbb{R}^{N}} a(x, u(x), \lambda) d \nu_{x}^{k}(\lambda)=a\left(x, u(x), \nabla T_{k}(u(x))\right) \text { a.e. } x \in \Omega .
$$


Now, by using Theorem 2.1 (ii), we deduce that $\nabla T_{k}\left(u_{n}\right)$ converges in measure to $\nabla T_{k}(u)$.

Lemma 3.6. $u$ is an entropy solution of (1.1).

\section{Proof of the Lemma 3.6,}

Let $u_{n}$ be a weak solution of the problem (3.2). Then, by Assertion $4-(d),\left(\nabla T_{k}\left(u_{n}\right)\right)_{n \in \mathbb{N}}$ converges to $\nabla T_{k}(u)$ in measure, thus (up to a subsequence still denoted $\left.\left(\nabla T_{k}\left(u_{n}\right)\right)_{n \in \mathbb{N}}\right),\left(\nabla T_{k}\left(u_{n}\right)\right)_{n \in \mathbb{N}}$ converges to $\nabla T_{k}(u)$ a.e. $\Omega$. Moreover, we deduce from Lemma 3.4 that $\nabla T_{k}\left(u_{n}\right)$ is uniformly bounded in $L^{p_{-}}(\Omega)$, so, $p_{-}-$equi-integrable on $\Omega$. Then, by using Vitali's Theorem

$$
\nabla T_{k}\left(u_{n}\right) \rightarrow \nabla T_{k}(u) \text { in } L^{p_{-}}(\Omega) \text {, which implies that } \nabla T_{k}\left(u_{n}\right) \rightarrow \nabla T_{k}(u) \text { in } L^{1}(\Omega) .
$$

Furthermore, thanks to Assertion 2, $u \in \mathcal{T}^{1, \pi(\cdot)}(\Omega)$ and it follows from Lemma 3.5 that

$$
u_{n} \rightarrow u \text { a.e on } \partial \Omega \text {. }
$$

Therefore, $u \in \mathcal{T}_{t r}^{1, \pi(.)}(\Omega)$. Now, using Lemma 3.2 the fact that $T_{n}\left(b\left(u_{n}\right)\right) \rightarrow b(u)$ a.e. in $\Omega$ and $u_{n} \rightarrow u$ a.e. on $\partial \Omega$, it follows from Fatou's Lemma that

$$
\int_{\Omega}|b(u)| \leq \liminf _{n \rightarrow+\infty} \int_{\Omega}\left|T_{n}\left(b\left(u_{n}\right)\right)\right| d x \leq\|f\|_{L^{1}(\Omega)}+\|g\|_{L^{1}(\partial \Omega)}
$$

and

$$
\int_{\partial \Omega}|u| \leq \liminf _{n \rightarrow+\infty} \int_{\partial \Omega}\left|T_{n}\left(u_{n}\right)\right| d x \leq \frac{1}{\lambda}\left(\|f\|_{L^{1}(\Omega)}+\|g\|_{L^{1}(\partial \Omega)}\right) .
$$

Hence, $b(u) \in L^{1}(\Omega)$ and $u \in L^{1}(\partial \Omega)$.

Let $\varphi \in \mathcal{C}^{\infty}(\bar{\Omega})$, then we can choose $T_{k}\left(u_{n}-\varphi\right)$ as a test function in (3.3) $\left(C^{\infty}(\bar{\Omega})\right.$ is dense in the space $W^{1, p_{+}}(\Omega)$ and $\left.T_{k}\left(u_{n}-\varphi\right) \in L^{\infty}(\partial \Omega)\right)$ to get

$$
\begin{aligned}
\int_{\Omega} T_{n}\left(b\left(u_{n}\right)\right) T_{k}\left(u_{n}-\varphi\right) d x & +\int_{\Omega} a\left(x, u_{n}, \nabla u_{n}\right) \cdot \nabla T_{k}\left(u_{n}-\varphi\right) d x+\int_{\partial \Omega} \lambda T_{n}\left(u_{n}\right) T_{k}\left(u_{n}-\varphi\right) d \sigma \\
& +\varepsilon \int_{\Omega}\left[\left|\nabla u_{n}\right|^{p_{+}-2} \nabla u_{n} \nabla T_{k}\left(u_{n}-\varphi\right)+\left|u_{n}\right|^{p_{+}-2} u_{n} T_{k}\left(u_{n}-\varphi\right)\right] d x \\
& =\int_{\Omega} f_{n} T_{k}\left(u_{n}-\varphi\right) d x+\int_{\partial \Omega} g_{n} T_{k}\left(u_{n}-\varphi\right) d \sigma
\end{aligned}
$$

For the first term of the left hand side of (3.43), we have

$$
\begin{aligned}
\int_{\Omega} T_{n}\left(b\left(u_{n}\right)\right) T_{k}\left(u_{n}-\varphi\right) d x & =\int_{\Omega}\left[T_{n}\left(b\left(u_{n}\right)\right)-T_{n}(b(\varphi))\right] T_{k}\left(u_{n}-\varphi\right) d x \\
& +\int_{\Omega} T_{n}(b(\varphi)) T_{k}\left(u_{n}-\varphi\right) d x
\end{aligned}
$$

By Fatou's Lemma, we infer

$$
\liminf _{n \rightarrow+\infty} \int_{\Omega} T_{n}\left(b\left(u_{n}\right)\right) T_{k}\left(u_{n}-\varphi\right) d x \geq \int_{\Omega} b(u) T_{k}(u-\varphi) d x
$$


since,

$$
\left[T_{n}\left(b\left(u_{n}\right)\right)-T_{n}(b(\varphi))\right] T_{k}\left(u_{n}-\varphi\right) \rightarrow\left(b(u-b(\varphi)) T_{k}(u-\varphi)\right. \text { a.e. }
$$

with

$$
\left[T_{n}\left(b\left(u_{n}\right)\right)-T_{n}(b(\varphi))\right] T_{k}\left(u_{n}-\varphi\right) \geq 0
$$

and

$$
T_{n}(b(\varphi)) T_{k}\left(u_{n}-\varphi\right) \rightarrow b(\varphi) T_{k}(u-\varphi) \text { in } L^{1}(\Omega) .
$$

In the same manner

$$
\liminf _{n \rightarrow+\infty} \lambda \int_{\partial \Omega} T_{n}\left(u_{n}\right) T_{k}\left(u_{n}-\varphi\right) d \sigma \geq \lambda \int_{\partial \Omega} u T_{k}(u-\varphi) d \sigma .
$$

For the fourth term of the left hand side of (3.43), we prove that

$$
\lim _{n \rightarrow+\infty} \varepsilon \int_{\Omega}\left[\left|\nabla u_{n}\right|^{p_{+}-2} \nabla u_{n} \nabla T_{k}\left(u_{n}-\varphi\right)+\left|u_{n}\right|^{p_{+}-2} u_{n} T_{k}\left(u_{n}-\varphi\right)\right] d x \geq 0 \text { as } \varepsilon \rightarrow 0 .
$$

Setting $l=k+\|\varphi\|_{L^{\infty}(\Omega)}$ we have,

$$
\begin{aligned}
& \varepsilon \int_{\Omega}\left|\nabla u_{n}\right|^{p_{+}-2} \nabla u_{n} \nabla T_{k}\left(u_{n}-\varphi\right) d x=\varepsilon \int_{\left\{\left|u_{n}-\varphi\right|<k\right\}}\left|\nabla T_{l}\left(u_{n}\right)\right|^{p_{+}-2} \nabla T_{l}\left(u_{n}\right) \nabla\left(T_{l}\left(u_{n}\right)-\varphi\right) d x \\
= & \varepsilon \int_{\left\{\left|u_{n}-\varphi\right|<k\right\}}\left|\nabla T_{l}\left(u_{n}\right)\right|^{p_{+}} d x-\varepsilon \int_{\left\{\left|u_{n}-\varphi\right|<k\right\}}\left|\nabla T_{l}\left(u_{n}\right)\right|^{p_{+}-2} \nabla T_{l}\left(u_{n}\right) \nabla \varphi d x \\
\geq & -\varepsilon \int_{\left\{\left|u_{n}-\varphi\right|<k\right\}}\left|\nabla T_{l}\left(u_{n}\right)\right|^{p_{+}-2} \nabla T_{l}\left(u_{n}\right) \nabla \varphi d x
\end{aligned}
$$

Moreover, by taking $v=T_{l}\left(u_{n}\right)$ in (3.3), we deduce that

$$
\varepsilon \int_{\Omega}\left[\left|\nabla u_{n}\right|^{p_{+}-2} \nabla u_{n} \nabla T_{l}\left(u_{n}\right)+\left|u_{n}\right|^{p_{+}-2} u_{n} T_{l}\left(u_{n}\right)\right] d x \leq l\left(\|f\|_{L^{1}(\Omega)}+\|g\|_{L^{1}(\partial \Omega)}\right),
$$

which implies that

$$
\varepsilon \int_{\Omega}\left|\nabla T_{l}\left(u_{n}\right)\right|^{p_{+}} d x \leq l\left(\|f\|_{L^{1}(\Omega)}+\|g\|_{L^{1}(\partial \Omega)}\right) .
$$

Therefore, $\varepsilon \nabla T_{l}\left(u_{n}\right)$ is uniformly bounded in $L^{p_{+}}(\Omega)$. From, Assertion $4-(d), \nabla T_{l}\left(u_{n}\right)$ converges a.e. in $\Omega$ (up to a subsequence) to $\nabla T_{l}(u)$. So, by Vitali's Theorem, $\varepsilon \nabla T_{l}\left(u_{n}\right)$ converges to $\varepsilon \nabla T_{l}(u)$ in $L^{p_{+}}(\Omega)$. Thus, $\varepsilon\left|\nabla T_{l}\left(u_{n}\right)\right|^{p_{+}-2} \nabla T_{l}\left(u_{n}\right) \chi_{\left\{\left|u_{n}-\varphi\right|<k\right\}}$ converges to $\varepsilon\left|\nabla T_{l}(u)\right|^{p_{+}-2} \nabla T_{l}(u) \chi_{\{|u-\varphi|<k\}}$ in $L^{p_{+}^{\prime}}(\Omega)$. Using (3.47), we obtain

$$
\lim _{n \rightarrow+\infty} \varepsilon \int_{\Omega}\left|\nabla u_{n}\right|^{p_{+}-2} \nabla u_{n} \nabla T_{k}\left(u_{n}-\varphi\right) d x \geq-\varepsilon \int_{\{|u-\varphi|<k\}}\left|\nabla T_{l}(u)\right|^{p_{+}-2} \nabla T_{l}(u) \nabla \varphi d x .
$$

Therefore,

$$
\lim _{n \rightarrow+\infty} \varepsilon \int_{\Omega}\left|\nabla u_{n}\right|^{p_{+}-2} \nabla u_{n} \nabla T_{k}\left(u_{n}-\varphi\right) d x \geq 0, \text { as } \varepsilon \rightarrow 0
$$


Now, we prove that

$$
\lim _{n \rightarrow+\infty} \varepsilon \int_{\Omega}\left|u_{n}\right|^{p_{+}-2} u_{n} T_{k}\left(u_{n}-\varphi\right) d x \geq 0, \text { as } \varepsilon \rightarrow 0 .
$$

We have

$$
\begin{aligned}
\int_{\Omega}\left|u_{n}\right|^{p_{+}-2} u_{n} T_{k}\left(u_{n}-\varphi\right) d x & =\int_{\Omega}\left(\left|u_{n}\right|^{p_{+}-2} u_{n}-|\varphi|^{p_{+}-2} \varphi\right) T_{k}\left(u_{n}-\varphi\right) d x \\
& +\int_{\Omega}|\varphi|^{p_{+}-2} \varphi T_{k}\left(u_{n}-\varphi\right) d x \\
& \geq \int_{\Omega}|\varphi|^{p_{+}-2} \varphi T_{k}\left(u_{n}-\varphi\right) d x
\end{aligned}
$$

since $\left(\left|u_{n}\right|^{p_{+}-2} u_{n}-|\varphi|^{p_{+}-2} \varphi\right) T_{k}\left(u_{n}-\varphi\right)$ is nonnegative.

Furthermore, $T_{k}\left(u_{n}-\varphi\right)$ converges weakly* to $T_{k}(u-\varphi)$ in $L^{\infty}(\Omega)$ and $|\varphi|^{p_{+}-2} \varphi \in L^{p_{+}^{\prime}}(\Omega)$, so

$$
\lim _{n \rightarrow+\infty} \int_{\Omega}|\varphi|^{p_{+}-2} \varphi T_{k}\left(u_{n}-\varphi\right) d x=\int_{\Omega}|\varphi|^{p_{+}-2} \varphi T_{k}(u-\varphi) d x .
$$

Combining (3.49) and (3.50), we obtain

$$
\lim _{n \rightarrow+\infty} \varepsilon \int_{\Omega}\left|u_{n}\right|^{p_{+}-2} u_{n} T_{k}\left(u_{n}-\varphi\right) d x \geq 0, \text { as } \varepsilon \rightarrow 0 .
$$

The relations (3.48) and (3.51) give us (3.46).

For the second term of the left hand side of (3.43), we recall that $l=k+\|\varphi\|_{L^{\infty}(\Omega)}$ and we get

$$
\begin{aligned}
& \int_{\Omega} a\left(x, u_{n}, \nabla u_{n}\right) \cdot \nabla T_{k}\left(u_{n}-\varphi\right) d x=\int_{\Omega} a\left(x, u_{n}, \nabla T_{l}\left(u_{n}\right)\right) \cdot \nabla\left(T_{l}\left(u_{n}\right)-\varphi\right) \chi_{\left\{\left|u_{n}-\varphi\right|<k\right\}} d x \\
= & \int_{\Omega} a\left(x, u_{n}, \nabla T_{l}\left(u_{n}\right)\right) \cdot \nabla T_{l}\left(u_{n}\right) \chi_{\left\{\left|u_{n}-\varphi\right|<k\right\}} d x-\int_{\Omega} a\left(x, u_{n}, \nabla T_{l}\left(u_{n}\right)\right) \cdot \nabla \varphi \chi_{\left\{\left|u_{n}-\varphi\right|<k\right\}} d x .
\end{aligned}
$$

Moreover, $a\left(x, u_{n}, \nabla T_{l}\left(u_{n}\right)\right) . \nabla T_{l}\left(u_{n}\right) \chi_{\left\{\left|u_{n}-\varphi\right|<k\right\}}$ is nonnegative and converges a.e. in $\Omega$ to $a\left(x, u, \nabla T_{l}(u)\right) \nabla T_{l}(u) \chi_{\{|u-\varphi|<k\}}$. Thanks to Fatou's Lemma, we get

$$
\liminf _{n \rightarrow+\infty} \int_{\Omega} a\left(x, u_{n}, \nabla T_{l}\left(u_{n}\right)\right) . \nabla T_{l}\left(u_{n}\right) \chi_{\left\{\left|u_{n}-\varphi\right|<k\right\}} d x \geq \int_{\Omega} a\left(x, u, \nabla T_{l}(u)\right) . \nabla T_{l}(u) \chi_{\{|u-\varphi|<k\}} d x .
$$

We now focus our attention on $\int_{\Omega} a\left(x, u_{n}, \nabla T_{l}\left(u_{n}\right)\right) \nabla \varphi \chi_{\left\{\left|u_{n}-\varphi\right|<k\right\}}$.

Let us prove that $a\left(x, u_{n}, \nabla T_{l}\left(u_{n}\right)\right) . \nabla \varphi \chi_{\left\{\left|u_{n}-\varphi\right|<k\right\}}$ is equi-integrable. Let $E$ be a subset of $\Omega$.

$$
\begin{aligned}
\int_{E} a\left(x, u_{n}, \nabla T_{l}\left(u_{n}\right)\right) . \nabla \varphi \chi_{\left\{\left|u_{n}-\varphi\right|<k\right\}} d x & \leq \int_{E}\left|a\left(x, u_{n}, \nabla T_{l}\left(u_{n}\right)\right)\right||\nabla \varphi| d x \\
& \leq \int_{E} \frac{1}{\pi_{n}^{\prime}(.)}\left|a\left(x, u_{n}, \nabla T_{l}\left(u_{n}\right)\right)\right|^{\pi_{n}^{\prime}(.)} d x+\int_{E} \frac{1}{\pi_{n}(.)}|\nabla \varphi|^{\pi_{n}(.)} d x \\
& \leq C_{1} \int_{E}\left(\mathcal{M}(x)+\left|\nabla T_{l}\left(u_{n}\right)\right|^{\pi_{n}(.)}\right) d x+\int_{E}|\nabla \varphi|^{\pi_{n}(.)} d x .
\end{aligned}
$$


Moreover,

$$
\begin{aligned}
\int_{E}|\nabla \varphi|^{\pi_{n}(.)} d x & =\int_{E \cap\{|\nabla \varphi| \leq 1\}}|\nabla \varphi|^{\pi_{n}(.)} d x+\int_{E \cap\{|\nabla \varphi|>1\}}|\nabla \varphi|^{\pi_{n}(.)} d x \\
& \leq \operatorname{meas}(E)+\int_{E}|\nabla \varphi|^{p_{+}} d x
\end{aligned}
$$

since $|\nabla \varphi|^{p_{+}}, \mathcal{M} \in L^{1}(\Omega)$ and $\left|\nabla T_{l}\left(u_{n}\right)\right|^{\pi_{n}(.)}$ is equi-integrable (using density argument for $C^{\infty}(\bar{\Omega})$ and (3.4)). Then, we obtain

$$
\lim _{\operatorname{meas}(E) \rightarrow 0} \int_{E} a\left(x, u_{n}, \nabla T_{l}\left(u_{n}\right)\right) \nabla \varphi \chi_{\left\{\left|u_{n}-\varphi\right|<k\right\}} d x=0 .
$$

Furthermore,

$$
a\left(x, u_{n}, \nabla T_{l}\left(u_{n}\right)\right) \nabla \varphi \chi_{\left\{\left|u_{n}-\varphi\right|<k\right\}} \rightarrow a\left(x, u, \nabla T_{l}(u)\right) . \nabla \varphi \chi_{\{|u-\varphi|<k\}} \quad \text { a.e. in } \quad \Omega .
$$

By applying Vitali's Theorem, we obtain

$$
\lim _{n \rightarrow+\infty} \int_{\Omega} a\left(x, u_{n}, \nabla T_{l}\left(u_{n}\right)\right) \nabla \varphi \chi_{\left\{\left|u_{n}-\varphi\right|<k\right\}} d x=\int_{\Omega} a\left(x, u, \nabla T_{l}(u)\right) . \nabla \varphi \chi_{\{|u-\varphi|<k\}} d x .
$$

Using (3.52), (3.53) and (3.54) we get

$$
\begin{aligned}
\liminf _{n \rightarrow+\infty} \int_{\Omega} a\left(x, u_{n}, \nabla u_{n}\right) . \nabla T_{k}\left(u_{n}-\varphi\right) d x & \geq \int_{\Omega} a\left(x, u, \nabla T_{l}(u)\right) \nabla\left(T_{l}(u)-\varphi\right) \chi_{\{|u-\varphi|<k\}} d x \\
& =\int_{\Omega} a(x, u, \nabla u) \nabla T_{k}(u-\varphi) d x .
\end{aligned}
$$

Now, we consider the right hand side of (3.43). For the first term of the right hand side of (3.43), since $f_{n} \rightarrow f$ in $L^{1}(\Omega)$ and $T_{k}\left(u_{n}-\varphi\right) \rightarrow^{*} T_{k}(u-\varphi)$ in $L^{\infty}(\Omega)$, we have

$$
\lim _{n \rightarrow+\infty} \int_{\Omega} f_{n} T_{k}\left(u_{n}-\varphi\right) d x=\int_{\Omega} f T_{k}(u-\varphi) d x
$$

For the second term of the right hand side of (3.43), by using the fact that $g_{n}$ strongly converges to $g$ in $L^{1}(\partial \Omega)$, we obtain

$$
\lim _{n \rightarrow+\infty} \int_{\partial \Omega} g_{n} T_{k}\left(u_{n}-\varphi\right) d \sigma=\int_{\partial \Omega} g T_{k}(u-\varphi) d \sigma,
$$

since

$$
T_{k}\left(u_{n}-\varphi\right) \rightarrow^{*} T_{k}(u-\varphi) \text { in } L^{\infty}(\partial \Omega),
$$

because $u_{n} \rightarrow u$ a.e. on $\partial \Omega$. 
Using (3.44), (3.45), (3.46), (3.55), (3.56), (3.57) and (3.43), we get

$$
\begin{aligned}
& \int_{\Omega} b(u) T_{k}(u-\varphi) d x+\int_{\Omega} a(x, u, \nabla u) \cdot \nabla T_{k}(u-\varphi) d x+\int_{\partial \Omega} \lambda u T_{k}(u-\varphi) d \sigma \\
\leq & \liminf _{n \rightarrow+\infty}\left(\int_{\Omega} T_{n}\left(b\left(u_{n}\right)\right) T_{k}\left(u_{n}-\varphi\right) d x+\int_{\Omega} a\left(x, u_{n}, \nabla u_{n}\right) \cdot \nabla T_{k}\left(u_{n}-\varphi\right) d x\right. \\
+ & \left.\int_{\partial \Omega} \lambda T_{n}\left(u_{n}\right) T_{k}\left(u_{n}-\varphi\right) d \sigma+\varepsilon \int_{\Omega}\left[\left|\nabla u_{n}\right|^{p_{+}-2} \nabla u_{n} \nabla T_{k}\left(u_{n}-\varphi\right)+\left|u_{n}\right|^{p+-2} u_{n} T_{k}\left(u_{n}-\varphi\right)\right] d x\right) \\
= & \int_{\Omega} f T_{k}(u-\varphi) d x+\int_{\partial \Omega} g T_{k}(u-\varphi) d \sigma, \text { as } \varepsilon \rightarrow 0,
\end{aligned}
$$

which is equivalent to

$$
\begin{aligned}
\int_{\Omega} b(u) T_{k}(u-\varphi) d x+\int_{\Omega} a(x, u, \nabla u) . \nabla T_{k}(u-\varphi) d x & +\int_{\partial \Omega} \lambda u T_{k}(u-\varphi) d \sigma \\
& \leq \int_{\Omega} f T_{k}(u-\varphi) d x+\int_{\partial \Omega} g T_{k}(u-\varphi) d \sigma,
\end{aligned}
$$

for $\varphi \in C^{\infty}(\bar{\Omega})$.

As $\pi($.$) verifies the log-Hlder condition (2.1) , C^{\infty}(\bar{\Omega})$ is dense in the space $W^{1, \pi(.)}(\Omega)$. Moreover, $W^{1, \pi(.)}(\Omega) \hookrightarrow W^{1, p_{-}}(\Omega) \hookrightarrow L^{\infty}(\Omega)$, since $\pi(.) \geq p_{-}>N$ and $\Omega$ is a bounded open domain with Lipschitz boundary $\partial \Omega$. Therefore, the inequality (3.59) holds true for $\varphi \in W^{1, \pi(.)}(\Omega) \cap L^{\infty}(\Omega)$. Hence, $u$ is an entropy solution of (1.1).

Now, we state the uniqueness result of entropy solution. This result uses the same arguments as [2]-Theorem 2.8 .

Theorem 3.5. Assume that $b$ is strictly increasing. Assume that $a=a(x, z, \eta)$ satisfies $\left(A_{3}\right)-\left(A_{6}\right)$ and $\mathcal{M}$ constant. Moreover, a satisfies:

for all bounded subset $K$ of $\mathbb{R} \times \mathbb{R}^{N}$, there exists a constant $C(K)$ such that

$$
\begin{aligned}
& \text { a.e. } x \in \Omega, \text { for all }(z, \eta),(\tilde{z}, \eta) \in K, \\
& |a(x, z, \eta)-a(x, \tilde{z}, \eta)| \leq C(K)|z-\tilde{z}| .
\end{aligned}
$$

Finally, suppose the following regularity property:

$$
\begin{aligned}
& \text { for all } f \in L^{\infty}(\Omega) \text { and } g \in L^{\infty}(\partial \Omega) \\
& \text { there exists an entropy solution of (1.1), } \\
& \text { which is Lipchitz continuous on } \bar{\Omega} \text {. }
\end{aligned}
$$

Then, for all $f \in L^{1}(\Omega)$ and $g \in L^{1}(\partial \Omega)$ the problem (1.1) admits a unique entropy solution. 
Remark 3.6. As in [2, Theorem 2.8], the condition (3.61) goes back to idea of [3]. Moreover, in the Theorem 3.5 the relation (3.60) is used to obtain the inequality (3.69) below.

Proof. The proof of this theorem is done in two steps.

Step 1. A priori estimates.

Lemma 3.7. If $v$ is an entropy solution of (1.1), there exists a positive constant $C$ such that

$$
\rho_{p(., v(.))}\left(|\nabla v| \chi_{F}\right) \leq C k,
$$

where $F=\{h-k<|v|<h\}, h>k>0$.

Proof. Let $\varphi=T_{h-k}(v)$ as test function in the entropy inequality (3.1), we get

$$
\begin{aligned}
\int_{\Omega} a(x, v, \nabla v) \cdot \nabla T_{k}\left(v-T_{h-k}(v)\right) d x & +\int_{\Omega} b(v) T_{k}\left(v-T_{h-k}(v)\right) d x+\lambda \int_{\partial \Omega} v T_{k}\left(v-T_{h-k}(v)\right) d \sigma \\
& \leq \int_{\Omega} f T_{k}\left(v-T_{h-k}(v)\right) d x+\int_{\partial \Omega} g T_{k}\left(v-T_{h-k}(v)\right) d \sigma .
\end{aligned}
$$

Thus,

$$
\int_{\{h-k<|v|<h\}} a(x, v, \nabla v) . \nabla T_{k}\left(v-T_{h-k}(v)\right) d x \leq k\left(\|f\|_{L^{1}(\Omega)}+\|g\|_{L^{1}(\partial \Omega)}\right)
$$

and using $\left(A_{6}\right)$, we have

$$
\int_{F}|\nabla v|^{p(x, v(x))} d x \leq k C_{2}\left(\|f\|_{L^{1}(\Omega)}+\|g\|_{L^{1}(\partial \Omega)}\right) .
$$

Consequently,

$$
\rho_{p(., v(.))}\left(|\nabla v| \chi_{F}\right) \leq C k, \text { where } C=C_{2}\left(\|f\|_{L^{1}(\Omega)}+\|g\|_{L^{1}(\partial \Omega)}\right) .
$$

We give the following lemma.

Lemma 3.8. If $u$ is an entropy solution of (1.1), then

$$
\operatorname{meas}(\{|u|>h\}) \leq \frac{\|f\|_{L^{1}(\Omega)}+\|g\|_{L^{1}(\partial \Omega)}}{\min (b(h),|b(-h)|)}, \forall h \geq 1 .
$$

Proof. Let us take $\varphi=0$ and $k=h$ in entropy inequality (3.1). Since

$$
\int_{\Omega} a(x, u, \nabla u) \cdot \nabla T_{h}(u) d x+\lambda \int_{\partial \Omega} u T_{h}(u) d \sigma \geq 0,
$$

the relation (3.1) gives

$$
\int_{\Omega} b(u) T_{h}(u) d x \leq \int_{\Omega} f T_{h}(u) d x+\int_{\partial \Omega} g T_{h}(u) d \sigma .
$$


Then,

$$
\int_{\{|u| \leq h\}} b(u) T_{h}(u) d x+\int_{\{|u|>h\}} b(u) T_{h}(u) d x \leq h\left(\|f\|_{L^{1}(\Omega)}+\|g\|_{L^{1}(\partial \Omega)}\right),
$$

or

$$
\int_{\{|u|>h\}} \frac{b(u) T_{h}(u)}{h} d x=\int_{\{u>h\}} b(u) d x+\int_{\{u<-h\}}-b(u) d x \leq\left(\|f\|_{L^{1}(\Omega)}+\|g\|_{L^{1}(\partial \Omega)}\right) .
$$

Therefore,

$$
\int_{\{|u|>h\}}|b(u)| d x \leq\|f\|_{L^{1}(\Omega)}+\|g\|_{L^{1}(\partial \Omega)}
$$

Since $b$ is nondecreasing, we deduce

$$
\int_{\{|u|>h\}} \min (b(h),|b(-h)|) d x \leq \int_{\{|u|>h\}}|b(u)| \leq\|f\|_{L^{1}(\Omega)}+\|g\|_{L^{1}(\partial \Omega)}, \quad \forall h \geq 1 .
$$

So,

$$
\operatorname{meas}(\{|u|>h\}) \leq \frac{\|f\|_{L^{1}(\Omega)}+\|g\|_{L^{1}(\partial \Omega)}}{\min (b(h),|b(-h)|)}, \forall h \geq 1
$$

Step 2. Uniqueness.

The existence has already been proved. Now, we show the uniqueness. For more details see [2]Proof of Theorem 2.8.

Let $u$ be a Lipschitz continuous entropy solution of (1.1) with $f \in L^{\infty}(\Omega), g \in L^{\infty}(\partial \Omega)$ and $v$ be an entropy solution, with $\hat{f} \in L^{1}(\Omega), \hat{g} \in L^{1}(\partial \Omega)$.

Since $\Omega$ is open bounded domain with smooth boundary $\partial \Omega$, the space of Lipschitz functions $C^{0,1}(\bar{\Omega})$ and $W^{1, \infty}(\Omega)$ are homeomorphic and they can be identified. Therefore, $u$ belongs to $W^{1, \infty}(\Omega)$. Thus, for all $h>0$, we can write the entropy inequality corresponding to the solution $u$, with $T_{h}(v)$ as a test function and to the solution $v$, with $T_{h}(u)$ as a test function. For all $k>0$, we get

$$
\left\{\begin{array}{l}
\int_{\Omega} a(x, u, \nabla u) \cdot \nabla T_{k}\left(u-T_{h}(v)\right) d x+\int_{\Omega} b(u) T_{k}\left(u-T_{h}(v)\right) d x \\
+\lambda \int_{\partial \Omega} u T_{k}\left(u-T_{h}(v)\right) d \sigma \leq \int_{\Omega} f T_{k}\left(u-T_{h}(v)\right) d x+\int_{\partial \Omega} g T_{k}\left(u-T_{h}(v)\right) d \sigma
\end{array}\right.
$$

and

$$
\left\{\begin{array}{l}
\int_{\Omega} a(x, v, \nabla v) \cdot \nabla T_{k}\left(v-T_{h}(u)\right) d x+\int_{\Omega} b(v) T_{k}\left(v-T_{h}(u)\right) d x \\
+\lambda \int_{\partial \Omega} v T_{k}\left(v-T_{h}(u)\right) d \sigma \leq \int_{\Omega} \hat{f} T_{k}\left(v-T_{h}(u)\right) d x+\int_{\partial \Omega} \hat{g} T_{k}\left(v-T_{h}(u)\right) d \sigma
\end{array}\right.
$$


Adding (3.62) and (3.63) we obtain

$$
\left\{\begin{array}{l}
\int_{\Omega} a(x, u, \nabla u) \cdot \nabla T_{k}\left(u-T_{h}(v)\right) d x+\int_{\Omega} a(x, v, \nabla v) . \nabla T_{k}\left(v-T_{h}(u)\right) d x \\
+\int_{\Omega} b(u) T_{k}\left(u-T_{h}(v)\right) d x+\int_{\Omega} b(v) T_{k}\left(v-T_{h}(u)\right) d x \\
+\lambda \int_{\partial \Omega} u T_{k}\left(u-T_{h}(v)\right) d \sigma+\lambda \int_{\partial \Omega} v T_{k}\left(v-T_{h}(u)\right) d \sigma \\
\leq \int_{\Omega}\left[f T_{k}\left(u-T_{h}(v)\right)+\hat{f} T_{k}\left(v-T_{h}(u)\right)\right] d x \\
+\int_{\partial \Omega}\left[g T_{k}\left(u-T_{h}(v)\right)+\hat{g} T_{k}\left(v-T_{h}(u)\right)\right] d \sigma .
\end{array}\right.
$$

Set $A=\{0<|u-v|<k,|v| \leq h\} ; B=A \cap\{|u| \leq h\} ; C=A \cap\{|u|>h\}$ and $A^{\prime}=\{0<|v-u|<k,|u| \leq h\} ; \quad B^{\prime}=A^{\prime} \cap\{|v| \leq h\} ; \quad C^{\prime}=A^{\prime} \cap\{|v|>h\}$. We start with the first integral in (3.64). We have

$$
\begin{aligned}
& \int_{\left\{0<\left|u-T_{h}(v)\right|<k\right\}} a(x, u, \nabla u) \cdot \nabla T_{k}\left(u-T_{h}(v)\right) d x \\
= & \int_{\left\{0<\left|u-T_{h}(v)\right|<k\right\} \cap\{|v| \leq h\}} a(x, u, \nabla u) \cdot \nabla T_{k}\left(u-T_{h}(v)\right) d x \\
+ & \int_{\left\{0<\left|u-T_{h}(v)\right|<k\right\} \cap\{|v|>h\}} a(x, u, \nabla u) \cdot \nabla T_{k}\left(u-T_{h}(v)\right) d x \\
= & \int_{\{0<|u-v|<k\} \cap\{|v| \leq h\}} a(x, u, \nabla u) \cdot \nabla(u-v) d x \\
+ & \int_{\{0<|u-h \operatorname{sign}(v)|<k\} \cap\{|v|>h\}} a(x, u, \nabla u) \cdot \nabla u d x \\
\geq & \int_{A} a(x, u, \nabla u) \nabla(u-v) d x \\
= & \int_{B} a(x, u, \nabla u) \nabla(u-v) d x+\int_{C} a(x, u, \nabla u) \nabla(u-v) d x .
\end{aligned}
$$

Then, we get

$$
\left\{\begin{array}{l}
\int_{\left\{0<\left|u-T_{h}(v)\right|<k\right\}} a(x, u, \nabla u) \cdot \nabla T_{k}\left(u-T_{h}(v)\right) d x \\
\geq \int_{B} a(x, u, \nabla u) \nabla(u-v) d x-\int_{C} a(x, u, \nabla u) \nabla v d x .
\end{array}\right.
$$

Now we use the fact that $\nabla u$ is bounded. By assumption of the theorem ( $\mathcal{M}$ is constant), $|a(x, u, \nabla u)| \leq C\left(|\nabla u|^{p(x, u(x))}+1\right) \in L^{\infty}(\Omega)$. Therefore, there exists a constant $K$ such that

$$
\begin{aligned}
\left|\int_{C} a(x, u, \nabla u) \nabla v d x\right| & \leq \int_{C}|a(x, u, \nabla u)||\nabla v| d x \\
& \leq K \int_{C}|\nabla v| d x \leq K \int_{\{h-k<|v|<h\}}|\nabla v| d x
\end{aligned}
$$

since $C \subset\{h-k<|v|<h\}$.

Thanks to Lemma 3.8 $\lim _{h \rightarrow+\infty} \operatorname{meas}(\{h-k<|v|<h\})=0$ and by Lemma 3.7, $|\nabla v| \chi_{F} \in L^{1}(\Omega)$. 
So, the right hand side of (3.66) converges to zero, as $h$ goes to infinity.

Consequently, the second integral of the right hand side of (3.65) converges to zero, as $h$ goes to infinity. Then, we can write that

$$
\begin{aligned}
\int_{\left\{0<\left|u-T_{h}(v)\right|<k\right\}} a(x, u, \nabla u) \cdot \nabla T_{k}\left(u-T_{h}(v)\right) d x & \geq \int_{B} a(x, u, \nabla u) \cdot \nabla(u-v) d x \\
& +I_{h}, \text { with } \lim _{h \rightarrow+\infty} I_{h}=0 .
\end{aligned}
$$

As $B=B^{\prime}$, we may adopt the same procedure to treat the second integral of (3.64) to obtain

$$
\begin{aligned}
\int_{\left\{0<\left|v-T_{h}(u)\right|<k\right\}} a(x, v, \nabla v) \cdot \nabla T_{k}\left(v-T_{h}(u)\right) d x & \geq-\int_{B} a(x, v, \nabla v) \cdot \nabla(u-v) d x \\
& +J_{h}, \text { with } \lim _{h \rightarrow+\infty} J_{h}=0 .
\end{aligned}
$$

For the other terms in the left hand side of (3.64), we denote by

$$
K_{h}=\int_{\Omega} b(u) T_{k}\left(u-T_{h}(v)\right) d x+\int_{\Omega} b(v) T_{k}\left(v-T_{h}(u)\right) d x
$$

and

$$
L_{h}=\lambda \int_{\partial \Omega} u T_{k}\left(u-T_{h}(v)\right) d \sigma+\lambda \int_{\partial \Omega} v T_{k}\left(v-T_{h}(u)\right) d \sigma .
$$

We have

$$
b(u) T_{k}\left(u-T_{h}(v)\right) \rightarrow b(u) T_{k}(u-v) \text { a.e. in } \Omega \text { as } h \rightarrow+\infty
$$

and

$$
\left|b(u) T_{k}\left(u-T_{h}(v)\right)\right| \leq k|b(u)| \in L^{1}(\Omega) .
$$

Then, by Lebesgue dominated convergence Theorem, we get

$$
\lim _{h \rightarrow+\infty} \int_{\Omega} b(u) T_{k}\left(u-T_{h}(v)\right) d x=\int_{\Omega} b(u) T_{k}(u-v) d x
$$

and

$$
\lim _{h \rightarrow+\infty} \int_{\Omega} b(v) T_{k}\left(v-T_{h}(u)\right) d x=\int_{\Omega} b(v) T_{k}(v-u) d x .
$$

Then,

$$
\lim _{h \rightarrow+\infty} K_{h}=\int_{\Omega}(b(u)-b(v)) T_{k}(u-v) d x .
$$

Similarly, we obtain

$$
\lim _{h \rightarrow+\infty} L_{h}=\lambda \int_{\partial \Omega}(u-v) T_{k}(u-v) d \sigma .
$$

Now, we consider the right hand side of (3.64), we have

$$
\lim _{h \rightarrow+\infty}\left[f T_{k}\left(u-T_{h}(v)\right)+\hat{f} T_{k}\left(v-T_{h}(u)\right)\right]=(f-\hat{f}) T_{k}(u-v) \text { a.e. in } \Omega
$$


and

$$
\left|f T_{k}\left(u-T_{h}(v)\right)+\hat{f} T_{k}\left(v-T_{h}(u)\right)\right| \leq k(|f|+|\hat{f}|) \in L^{1}(\Omega) .
$$

By Lebesgue dominated convergence Theorem, we get

$$
\lim _{h \rightarrow+\infty} \int_{\Omega} f\left[T_{k}\left(u-T_{h}(v)\right)+T_{k}\left(v-T_{h}(u)\right)\right] d x=\int_{\Omega}(f-\hat{f}) T_{k}(u-v) d x .
$$

Similarly, we have

$$
\lim _{h \rightarrow+\infty} \int_{\partial \Omega} g\left[T_{k}\left(u-T_{h}(v)\right)+\hat{g} T_{k}\left(v-T_{h}(u)\right)\right] d \sigma=\int_{\partial \Omega}(g-\hat{g}) T_{k}(u-v) d \sigma .
$$

After passing to the limit as $h$ goes to $+\infty$ in (3.64), we get

$$
\left\{\begin{array}{l}
\int_{\{0<|u-v|<k\}}(a(x, u, \nabla u)-a(x, v, \nabla v)) \nabla(u-v) d x \\
+\int_{\Omega}(b(u)-b(v)) T_{k}(u-v) d x+\lambda \int_{\partial \Omega}(u-v) T_{k}(u-v) d \sigma \\
\leq \int_{\Omega}(f-\hat{f}) T_{k}(u-v) d x+\int_{\partial \Omega}(g-\hat{g}) T_{k}(u-v) d \sigma
\end{array}\right.
$$

which is equivalent to

$$
\left\{\begin{array}{l}
\int_{\{0<|u-v|<k\}}(a(x, u, \nabla v)-a(x, v, \nabla v)) \nabla(u-v) d x \\
+\int_{\{0<|u-v|<k\}}(a(x, u, \nabla u)-a(x, u, \nabla v)) \nabla(u-v) d x \\
+\int_{\Omega}(b(u)-b(v)) T_{k}(u-v) d x+\lambda \int_{\partial \Omega}(u-v) T_{k}(u-v) d \sigma \\
\leq \int_{\Omega}(f-\hat{f}) T_{k}(u-v) d x+\int_{\partial \Omega}(g-\hat{g}) T_{k}(u-v) d \sigma .
\end{array}\right.
$$

Dividing (3.68) by $k$ and letting $k$ goes to 0 , we have

$$
\left\{\begin{array}{l}
\lim _{k \rightarrow 0} \frac{1}{k} \int_{\{0<|u-v|<k\}}(a(x, u, \nabla v)-a(x, v, \nabla v)) \nabla(u-v) d x \\
+\lim _{k \rightarrow 0} \frac{1}{k} \int_{\{0<|u-v|<k\}}(a(x, u, \nabla u)-a(x, u, \nabla v)) \nabla(u-v) d x \\
+\int_{\Omega}|b(u)-b(v)| d x+\lambda \int_{\partial \Omega}|u-v| d \sigma \\
\leq \int_{\Omega}(f-\hat{f}) \operatorname{sign}(u-v) d x+\int_{\partial \Omega}(g-\hat{g}) \operatorname{sign}(u-v) d \sigma .
\end{array}\right.
$$

Thanks to the relation (3.60), the first integral of (3.69) goes to 0 as $k \rightarrow 0$ (See [2], proof of Theorem 2.8-Step 2). Thus, we obtain

$$
\left\{\begin{array}{l}
\lim _{k \rightarrow 0} \frac{1}{k} \int_{\{0<|u-v|<k\}}(a(x, u, \nabla u)-a(x, u, \nabla v)) \nabla(u-v) d x \\
+\int_{\Omega}|b(u)-b(v)| d x+\lambda \int_{\partial \Omega}|u-v| d \sigma \\
\leq \int_{\Omega}|f-\hat{f}| d x+\int_{\partial \Omega}|g-\hat{g}| d \sigma .
\end{array}\right.
$$


Since, the three integral of left-hand in (3.70) are positive, we deduce that

$$
\int_{\Omega}|b(u)-b(v)| d x+\lambda \int_{\partial \Omega}|u-v| d \sigma \leq \int_{\Omega}|f-\hat{f}| d x+\int_{\partial \Omega}|g-\hat{g}| d \sigma .
$$

Let us take a sequence $\left(f_{i}\right)_{i \in \mathbb{N}} \subset L^{\infty}(\Omega)$ and $\left(g_{i}\right)_{i \in \mathbb{N}} \subset L^{\infty}(\partial \Omega)$ and let $\left(u_{i}\right)_{i \in \mathbb{N}}$ be the corresponding sequence of Lipschitz continuous entropy solutions. By (3.71), we have

$$
\begin{aligned}
\int_{\Omega}|b(u)-b(v)| d x & +\lambda \int_{\partial \Omega}|u-v| d \sigma \leq \int_{\Omega}\left[\left|b(u)-b\left(u_{i}\right)\right|+\left|b(v)-b\left(u_{i}\right)\right|\right] d x \\
& +\lambda \int_{\partial \Omega}\left[\left|u-u_{i}\right|+\left|v-u_{i}\right|\right] d \sigma \\
& \leq \int_{\Omega}\left[\left|f-f_{i}\right|+\left|\hat{f}-f_{i}\right|\right] d x+\int_{\partial \Omega}\left[\left|g-g_{i}\right|+\left|\hat{g}-g_{i}\right|\right] d \sigma
\end{aligned}
$$

so that at the limit as $i \rightarrow \infty$ in left hand-side of (3.72) and using the density argument $\left(L^{\infty}(\Omega)\right.$ and $L^{\infty}(\partial \Omega)$ are dense (respectively) in $L^{1}(\Omega)$ and $\left.L^{1}(\partial \Omega)\right)$, we infer that

$$
b(u)=b(v) \text { a.e. in } \Omega \text { and } u=v \text { a.e. on } \partial \Omega \text {. }
$$

Hence,

$$
u=v \text { a.e. in } \Omega \text { and } u=v \text { a.e. on } \partial \Omega \text {. }
$$

Since $b$ is assumed strictly increasing. 


\section{References}

[1] B. Andreianov, M. Bendahmane, S. Ouaro; Structural stability for variable exponent elliptic problems. I. The $p(x)$-Laplacian kind problems, Nonlinear Analysis, 73 (2010), $2-24$.

[2] B. Andreianov, M. Bendahmane, S. Ouaro; Structural stability for variable exponent elliptic problems, II: The $p(u)$-Laplacian and coupled problems, Nonlinear Analysis, 72 (2010), $4649-4660$.

[3] B. Andreianov, F. Bouhsiss; Uniqueness for an elliptic parabolic problem with Neumann boundary condition, J. Evol. Equ. 4 (2) (2004) 273-295.

[4] F. Andreu, N. Igbida, J.M. Mazn, J. Toledo; $L^{1}$ existence and uniqueness results for quasi-linear elliptic equations with nonlinear boundary conditions, Ann. Inst. Henri Poincar 24 (2007), $61-89$.

[5] F. Andreu, J.M. Mazón, S. Segura de León, J. Toledo; Quasi-linear elliptic and parabolic equations in $L^{1}$ with nonlinear boundary conditions, Adv. Math. Sci. Appl. 7, No. 1 (1997), $183-213$.

[6] S.N. Antontsev, J.F. Rodrigues; On stationary thermo-rheological viscous flows, Ann. Univ. Ferrara Sez. VII Sci. Mat. 52 (1) (2006), 19 - 36.

[7] J.M. Ball; A version of the fundamental theorem for Young measures. PDEs and continum models of phase transitions (Nice, 1988), 207-215, Lecture Notes in Phys., 344, Springer, 1989.

[8] M.B. Benboubker, S. Ouaro, U. Traor; Entropy solutions for nonhomogeneous Neumann problems involving the generalized $p(x)$-laplacian operators and measure data, Nonlinear Evolution Equation and Application. Volume 2014, Number 5, pp. 53 - 76.

[9] Y. Chen, S. Levine, M. Rao; Variable exponent, linear growth functionals in image restoration. SIAM. J.Appl. Math., 66 (2006), 1383 - 1406.

[10] G. Dolzmann, N. Hungerbhler, S. Mller; The p-harmonic system with measure-valued right hand side, Ann. Inst. H. Poincar. Anal. Non Linaire 14 (3) (1997), 353 - 364.

[11] R. Eymard, T. Galloet and R. Herbin; Finite volume methods. Handbook of numerical analysis, Vol. VII, 713 - 1020, North-Holland, 2000.

[12] N. Hungerbhler; Quasi-linear parabolic systems in divergence form with weak monotonicity, Duke Math. J. 107 (3) (2001), 497 - 520. 
[13] N. Hungerbhler; A refinement of Ball's theorem on Young measures. New York J. Math. 3 (1997), $48-53$.

[14] I. Ibrango, S. Ouaro; Entropy solution for doubly nonlinear elliptic anisotropic problems with Fourier boundary conditions, Differential Inlusions, Control and Optimization, vol 35 (2015), $123-150$.

[15] I. Nyanquini, S. Ouaro; Entropy solution for nonlinear elliptic problem involving variable exponent and Fourier type boundary condition, Afr. Mat. 23, No. 2, 205 - 228 (2012).

[16] V. K. Le, On sub-supersolution method for variational inequalities with Leray-Lions operators in variable exponent spaces. Nonlinear Anal. 71 (2009), 3305 - 3321.

[17] J.-L. Lions; Quelques mthodes de rsolution de problmes aux limites nonlinaires, Dunod. Gauthier-Villars, Paris, 1969.

[18] S. Ouaro, A Tchousso; Well-posedness result for a nonlinear elliptic problem involving variable exponent and Robin type boundary condition, African Diaspora Journal of Mathematics 11, No. 2, 36 - 64 (2011).

[19] P. Pedregal; Parametrized measures and variational principles. Progress in Nonlinear Differential Equations and their Applications, 30. Birkhäuser, Basel, 1997.

[20] M. Ruzicka; Electrorheological fluids: modelling and mathematical theory. Lecture Notes in Mathematics 1748, Springer-Verlag, Berlin, 2002.

[21] M. Sanchón, J. M. Urbano; Entropy solutions for the $p(x)$-Laplace Equation, Trans. Amer. Math. Soc. 361 (2009), no. 12, 6387 - 6405.

[22] R. E. Showalter, Monotone operators in Banach space and nonlinear partial differential equations, Mathematical surveys and monographs, 49, American Mathematical Society.

[23] L. Wang, Y. Fan, W. Ge; Existence and multiplicity of solutions for a Neumann problem involving the $p(x)$-Laplace operator. Nonlinear Anal. 71 (2009), $4259-4270$.

[24] J. Yao; Solutions for Neumann boundary value problems involving $p(x)$-Laplace operator. Nonlinear Anal: (TMA) 68(2008), $1271-1283$. 